\title{
Virtuelle Entkopplung von fachlichen und IT- Strukturen für das IT/Business Alignment - Grundlagen, Architekturgestaltung und Umsetzung am Beispiel der Domänenbildung
}

\author{
Die Integration von Business und IT ist eines der Kernthemen der Wirtschaftsinfor- \\ matik. Der gegenseitige Abgleich von fachlichen und IT- Strukturen stellt dabei eine \\ zentrale Herausforderung dar. Für die Alignment-Architektur werden die elementaren \\ Artefakte fachliche Services, Applikationen und Domänen beschrieben. Die Elemente \\ der Alignment-Architektur haben weder eine direkte Entsprechung auf Business- noch \\ auf IT-Seite. Sie sollten gröber sein als die Artefakte, welche sie verknüpfen. Existierende \\ Methoden können in den vorgeschlagenen Ansatz integriert werden. Alternativ dazu \\ wird ein Bottom-up-Ansatz vorgestellt und anhand einer Fallstudie für die Bildung von \\ Domänen als virtuelle, aggregierte Artefakte der Alignment-Architektur demonstriert.
}

DOI 10.1007/s11576-008-0115-0

\section{Die Autoren}

Dr.-Ing. Stephan Aier

Prof. Dr. Robert Winter

Institut für Wirtschaftsinformatik

Universität St. Gallen

Müller-Friedberg-Strasse 8

9000 St. Gallen

Schweiz

\{stephan.aier|robert.winter\}@unisg.ch

Eingereicht: 2008-05-01

Angenommen: 2008-09-02

Annahme nach zwei Überarbeitungen durch Prof. Dr. Buhl.

This article is also available in English via http://www.springerlink.com and http://www.bise-journal.org: Aier S, Winter R (2008) Virtual Decoupling for IT/Business Alignment - Conceptual Foundations, Architecture Design and Implementation Example. Bus Inf Syst Eng. doi:10.1007/s12599-008-0010-7.

\section{Einleitung}

Ihre Positionierung „Zwischen“ Betriebswirtschaftslehre und Informatik prädestiniert die Wirtschaftsinformatik für Integrationsfragestellungen. Integration wird oft als das Ur- und Hauptthema der
Wirtschaftsinformatik angesehen (Rosemann 1999; Vogler 2003). Integration ist ein komplexes Konzept, das durch die verschiedensten Ausprägungen hinsichtlich einer Vielzahl von Dimensionen gekennzeichnet wird. Häufig genannte Dimensionen sind

- Integrationsform (vereinigende vs. verknüpfende Integration),

- Integrationsreichweite (bereichsweite, unternehmensweite vs. unternehmensübergreifende Integration),

- Integrationsgegenstand (Daten-, Funktions-, Prozess-, Methoden- vs. Programmintegration) oder

- Integrationsrichtung (vertikale vs. horizontale Integration).

Eine ausführliche Übersicht über die phänomenologische Systematisierung von Integration findet sich u. a. in (Mertens 2007, S. 1-10).

Unter den verschiedenen Integrationsausprägungen kommt der Integration zwischen fachlichen Architekturen und IT-Architekturen besondere Bedeutung zu. „IT/Business Alignment“ stellt seit mehreren Jahren über alle Unternehmens-Größenklassen und über alle Branchen hinweg das Hauptthema der CIOs dar (Luftman 2005). IT/Business Alignment bedeutet allerdings nicht nur, dass IT-Architekturen an fachlichen Architekturen auszurichten sind. Stellt man einerseits „Strategie“ (Was-Fragen) und „Pro-
zesse/Infrastrukturen“ (Wie-Fragen) auf einer vertikalen Achse und andererseits „Fachliche Sicht“ und „IT-Sicht“ auf einer horizontalen Achse dar, können zwischen den sich ergebenden vier Quadranten „fachliche Strategie“, „IT-Strategie“, „fachliche Prozesse/Infrastrukturen“ und „IT-Prozesse/-Infrastrukturen“ mindestens vier Integrationsperspektiven unterscheiden werden (Henderson $u$. Venkatraman 1993).

Integration kann durch Vereinigen oder durch Verknüpfen erreicht werden (Rosemann 1999, S. 5 f). Zwar wirkt vereinigende Integration komplexitätsreduzierend, ist aber häufig nicht möglich oder nicht sinnvoll. Wenn z. B. Informationssysteme in unterschiedlichen Unternehmen einen unternehmensübergreifenden Geschäftsprozess unterstützen, ist vereinigende Integration aufgrund unterschiedlicher Verantwortlichkeiten und/ oder rechtlicher Rahmenbedingungen nicht möglich. Wenn z. B. unterschiedliche Artefakttypen wie fachliche Anforderungen auf der einen Seite und durch IT realisierte Funktionalitäten auf der anderen Seite abgestimmt werden müssen, ist vereinigende Integration ebenfalls nicht möglich. Selbst wenn vereinigende Integration möglich ist, ist sie nicht immer sinnvoll, um z. B. das Entstehen monolithischer, schwer wartbarer Informationssysteme zu vermeiden. 
Wenn eine vereinigende Integration nicht möglich oder sinnvoll ist, muss Integration durch Verknüpfung erfolgen. Für Integration durch Verknüpfung stellen Zuordnungsmechanismen ein zentrales Umsetzungsinstrument dar. Im IT/Business Alignment sind dann z. B. Mechanismen $\mathrm{zu}$ spezifizieren, die fachlichstrategische Artefakte (z. B. Leistungen, Ziele) zunächst fachlichen Umsetzungsartefakten (z. B. Prozesse, Organisationsstrukturen) und schließlich auch ITUmsetzungsartefakten (z. B. Softwarekomponenten, Datenstrukturen) zuordnen.

Es liegt zunächst nahe, die zu verknüpfenden Artefakte durch direkte Punkt-zuPunkt-Zuordnungen zu integrieren. Für komplexe Integrationssachverhalte darf erwartet werden, dass jedes der beteiligten Artefakte dann an mehreren solcher Zuordnungen beteiligt ist. Änderungen in solchen durch eine Vielzahl von Punktzu-Punkt-Zuordnungen verknüpften, komplexen Artefaktstrukturen haben die Tendenz, sich explosionsartig ,auszubreiten“. Ein Beispiel für dieses Problem ist die Verknüpfung von Dateistrukturen und Verarbeitungslogik in der COBOL-Programmierung. Die Änderung der Verarbeitungslogik kann eine Veränderung der „gelesenen“ Dateistruktur erfordern. Als Konsequenz muss nicht nur das Programm angepasst werden, das diese Datei „schreibt“. Auch alle anderen Programme müssen angepasst werden, die diese Datei verarbeiten.

Mit zunehmender Anzahl von Verknüpfungen, zunehmender Komplexität der zu verknüpfenden Artefaktstrukturen und zunehmender Änderungsdynamik des Gesamtsystems werden direkte Punktzu-Punkt-Zuordnungen immer ineffizienter. Die Lösung dieses Problems sind indirekte Zuordnungen mit Hilfe eines m:n-fähigen, flexiblen Zuordnungsmechanismus („Hub“). Das Konzept „Enterprise Application Integration“ (Kaib 2002; Linthicum 2000) stellt ein gutes Beispiel für einen solchen Zuordnungsmechanismus dar. An die Stelle einer Vielzahl von direkten Punkt-zu-Punkt-Verknüpfungen zwischen allen beteiligten Softwaresystemen tritt ein Zuordnungssystem, zu dem alle beteiligten Softwaresysteme nur eine einzige Verknüpfung (den sog. „Adapter“) haben. Eine u. U. sehr hohe Zahl direkter Punkt-zu-Punkt-Verknüpfungen wird durch eine weitaus geringere $Z$ ahl von Adaptern abgelöst.
Enterprise Application Integration verknüpft jedoch ausschließlich Softwaresysteme. Generell ist eine „Hub-and-Spoke“Architektur auf die verknüpfende Integration von Artefakten des gleichen Artefakttyps beschränkt. Zur Unterstützung von IT/Business Alignment müssen aber z. B. Aktivitäten aus Prozessen auf der einen Seite mit durch Softwarekomponenten realisierten Funktionalitäten auf der anderen Seite verknüpft werden - mithin also Artefakte unterschiedlicher Typen. Es ist anzustreben, auch diese Form der verknüpfenden Integration durch indirekte, m:n-fähige, flexible Zuordnungsmechanismen umzusetzen. Ein solcher Zuordnungsmechanismus ist dann architektonisch „Zwischen“ fachlichen Strukturen und IT-Strukturen positioniert.

Die Ziele dieses Beitrags sind,

1. zu zeigen, dass IT/Business Alignment durch eine verknüpfende Integration mit Hilfe eines indirekten, m:n-fähigen Zuordnungsmechanismus unterstützt werden kann, wodurch erhebliche Vorteile gegenüber direkten Punkt-zuPunkt-Zuordnungen realisiert werden können,

2. die Architektur dieses Zuordnungsmechanismus aus analogen Mechanismen abzuleiten, die in Systemtheorie, Informatik und Ökonomie entwickelt wurden,

3. für die Modellierung von IT/Business Alignment geeignete Zuordnungsartefakttypen vorzuschlagen, die eine indirekte, m:n-fähige Zuordnung von fachlichen Architekturen auf der einen Seite und IT-Architekturen auf der anderen Seite ermöglichen,

4. zu zeigen, dass neben einem Topdown-Entwurf dieser Zuordnungsartefakte auch Bottom-up-Vorgehensweisen sinnvoll sind und schließlich

5. an einem konkreten Anwendungsbeispiel, der Identifikation von Domänen in einem Telekommunikations-Ausrüstungsunternehmen, die Durchführbarkeit des Bottom-up-Entwurfs von Zuordnungsartefakten zu zeigen und den Beitrag der entwickelten Zuordnungsarchitektur nachzuweisen.

Die Strukturierung des Beitrags lehnt sich eng an das Prozessmodell gestaltungsorientierter Forschung für Informationssysteme an (Rossi u. Sein 2003):

- „Identify a need“: Zunächst wird die Notwendigkeit der Etablierung eines indirekten, m:n-fähigen Zuordnungsmechanismus „zwischen“ fachlichen Architek- turen auf der einen Seite und IT-Architekturen auf der anderen Seite begründet (Abschnitt 1). In aller Kürze wird ein terminologischer Rahmen umrissen, der das Verständnis der Autoren von Architekturen und Artefakttypen im IT/Business Alignment dokumentiert (Abschnitt 2). Das verfügbare Wissen zu m:n-fähigen Zuordnungsmechanismen in Systemtheorie, Informatik und Ökonomie wird diskutiert (Abschnitt 3). Auf dieser Grundlage wird als Gestaltungsziel die Übertragung bestehender Zuordnungsmechanismen auf das IT/ Business Alignment konkretisiert.

- „Build“: Bestehende indirekte, m:nfähige Zuordnungsmechanismen wie die Dreiebenen-Datenbankarchitektur oder der Marktmechanismus für standardisierte Güter werden auf die Integration fachlicher Architekturen und ITArchitekturen übertragen. Die dafür zu repräsentierenden Artefakte werden spezifiziert (Abschnitt 4). Schließlich wird gezeigt, dass Gestaltungsansätze für diese Artefakte meist zerlegend sind. Als Ergänzung wird ein Bottom-up-Gestaltungsansatz vorgeschlagen (Abschnitt $5)$.

- „Evaluate“ (Abschnitt 6): Die Bottomup-Identifikation von Zuordnungsartefakten wird am Beispiel der Domänenbildung prototypisch realisiert und auf ein reales Gestaltungsproblem angewandt. Die Anwendbarkeit des Teilkonzepts wird im Lichte des Gestaltungsziels (aus den Abschnitten 1 und 2) analysiert.

- „Learn \& Theorize“ (Abschnitt 7): Die Evaluations(teil)ergebnisse sowie die Implikationen des vorgeschlagenen Ansatzes werden diskutiert. Hinsichtlich der Implikationen wird insbesondere das Integrationsmanagement betrachtet.

\section{Terminologische Einordnung}

Leistungsbeschreibungen, Zieldefinitionen, Prozessspezifikationen, Organisationsstrukturen usw. sind fachliche Artefakte, die durch fachliche Architekturen abgebildet werden. Softwarekomponenten, Datenstrukturen usw. sind IT-Artefakte, die durch IT-Architekturen abgebildet werden. Im Gegensatz zu diesen Artefakten ist der oben skizzierte Zuordnungsmechanismus für IT/Business Alignment ein virtuelles Artefakt, da er nur zur „Übersetzung“ der verknüpften 
Strukturen dient und weder ein Teil fachlicher Architekturen noch ein Teil von IT-Architekturen ist.

Die elementaren Artefakte, mit deren Hilfe ein derartiger Zuordnungsmechanismus konstruiert werden kann, sind fachliche Services. Fachliche Services ordnen als Zuordnungsartefakte

- IT-Funktionalitäten (d. h. durch Softwarekomponenten und Datenstrukturen realisierte, elementare Anwendungssystemfunktionalitäten) auf der einen Seite

- Aktivitäten (d. h. für die Prozessausführung erforderliche, in Prozessen angeordnete, durch Anwendungssysteme unterstützte fachliche Aufgabenbestandteile) auf der anderen Seite indirekt und m:n-fähig zu. Fachliche Services müssen gröber granular sein als die verknüpften IT-Funktionalitäten und Aktivitäten, damit die Vorteile der (im Vergleich zur direkten Punkt-zu-Punkt-Verknüpfung) flexibleren Zuordnung nicht durch die zusätzliche Komplexität einer weiteren Architekturebene überwogen werden. Um das Top-down-Verständnis und die Kommunikation der Zuordnungsarchitektur zu unterstützen, werden in der Praxis zusätzlich zu fachlichen Services, den elementaren Zuordnungs-Artefakten, auch Applikationen und Domänen genutzt. Auch Applikationen und Domänen sind virtuelle Artefakte, da sie dem IT/Business Alignment dienen und weder ein Teil fachlicher Architekturen noch ein Teil von IT-Architekturen sind.

Die Architekturebene, auf der fachliche Services, Applikationen und Domänen repräsentiert werden, wird aufgrund ihres primären Zwecks als Alignment-Ebene bezeichnet. Ihre Artefakte heißen Alignment-Artefakte und bilden die Alignment-Architektur. Die Alignment-Ebene ist zusätzlich zur Ebene der fachlichen Architekturen (zur Repräsentation von Leistungen, Zielen, Prozessen, Organisationsstrukturen usw.) und zur Ebene der IT-Architekturen (zur Repräsentation von Softwarekomponenten, Datenstrukturen usw.) einzuführen, um die Integrationserfordernisse des IT/Business Alignment zu unterstützen. Da die auf Alignment-Ebene repräsentierten Artefakte virtuell sind, resultieren für die Gestaltung und das Management der Alignment-Ebene spezifische Herausforderungen. Auch wenn die Alignment-Architektur ausschließlich durch virtuelle Artefakte repräsen-
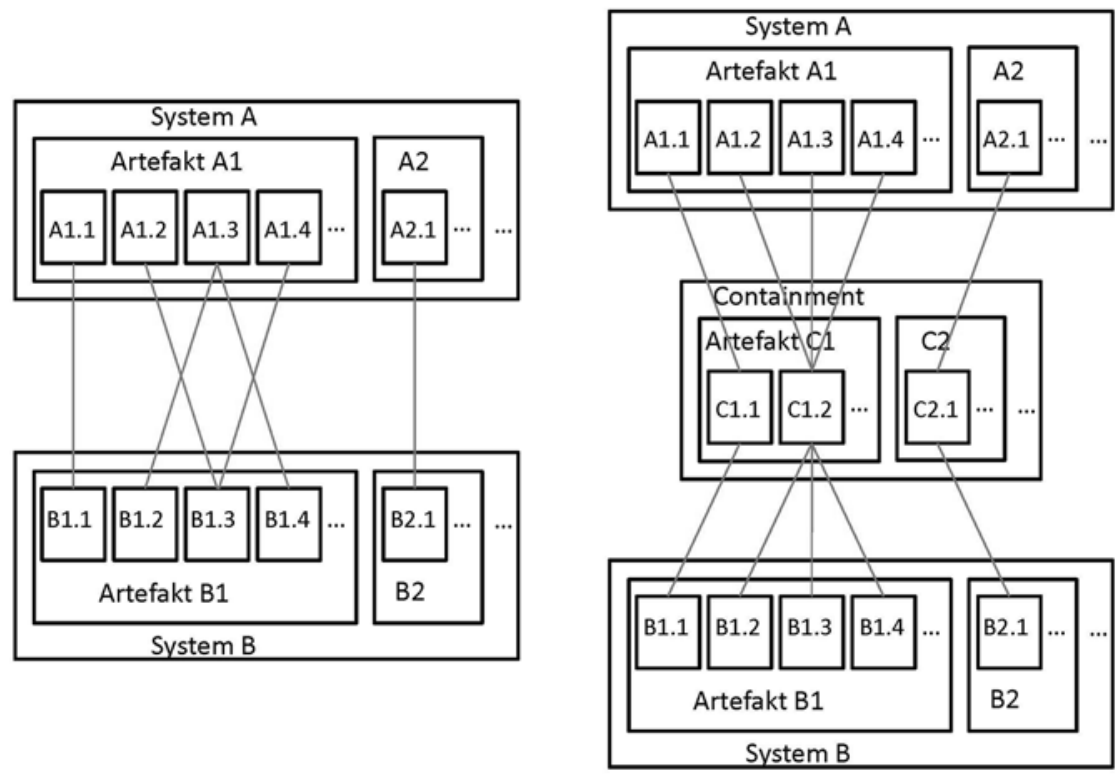

Abb. 1 Generischer, entkoppelnder Zuordnungsmechanismus in der Systemtheorie

tiert wird, tritt sie gleichwertig neben fachliche Architekturen und IT-Architekturen. Während z. B. fachliche Architekturen an fachlichen Gestaltungszielen wie Prozesseffektivität und -effizienz orientiert sind (Winter 2008) und während IT-Architekturen an technischen Gestaltungszielen wie Wiederverwendung, Skalierbarkeit und Performanz orientiert sind, werden für die Alignment-Architektur eigenständige Gestaltungsziele wie z. B. Transparenzschaffung, (verknüpfende) Integration, Vereinfachung und/ oder Flexibilisierung verfolgt (Winter $u$. Schelp 2006).

\section{Entkoppelnde Zuordnungs- mechanismen in Systemtheorie, Informatik und Ökonomie}

Im Folgenden wird das grundsätzliche Funktionsprinzip des Zuordnungsmechanismus anhand des Vokabulars der Systemtheorie allgemein beschrieben. Anschließend werden darauf aufbauend Beispiele aus der Informatik sowie der Ökonomie analysiert.

\subsection{Entkoppelnde Zuordnungsmecha- nismen in der Systemtheorie}

Der Ausgangspunkt für die Entkopplung in der Systemtheorie ist das Problem der Komplexität. Die Komplexität der Umwelt ist immer größer als die Komplexität des betrachteten Systems. Das System hat keine ausreichende Kapazität, um für jeden
Zustand der Umwelt einen angemessenen eigenen Zustand zu definieren, da es nicht in der Lage ist, für alle Umweltelemente direkte Punkt-zu-Punkt-Beziehungen aufzubauen. Die Möglichkeiten eines Systems, mit diesem Komplexitätsgefälle umzugehen, sind zum einen Ignoranz, d. h. nicht auf Umweltveränderungen $\mathrm{zu}$ reagieren, und zum anderen Generalisierung, d. h. auf unterschiedliche Veränderungen der Umwelt in gleicher Art und Weise zu reagieren.

Die Generalisierung unterbricht die direkten Punkt-zu-Punkt-Zuordnungen durch Bündelungen, die im System oder in der Umwelt vorgesehen werden können. Die Bündelung wird in der Systemtheorie unter dem Begriff der Stufentheorie diskutiert (Luhmann 2002, S. 169). Ashby nennt Systeme, die solche Stufenfunktionen aufweisen, ultrastabil (Ashby 1981, S. 48). Ultrastabile Systeme fangen Störungen der Umwelt lokal ab, ohne dass die Störungen auf das gesamte System wirken können. Stufenfunktionen stellen eine Interdependenzunterbrechung dar. Diese Interdependenzunterbrechung wird auch als "loose coupling“ (Glassman 1973; Weick 1976) bezeichnet (Luhmann 2002, S. 171). Das Gegenteil von "loose coupling" ist "tight coupling“ und kommt in natürlichen Systemen für gewöhnlich nicht vor.

Je höher der Umfang ist, in welchem ein System "tight coupling" implementiert, desto gefährdeter ist es, da Störungen, die auf Systemteile wirken, das gesamte System beeinflussen. Dies wurde vor allem 
in der Diskussion um Hochtechnologie als problematisch charakterisiert, da solche Systeme oft "tight coupling“ implementieren (Luhmann 1991; Perrow 1999). Die Antwort der Systemtheorie ist der Bau einer Schutzhülle, eines Containments, welche selbst wieder auf den Prinzipien des ,loose coupling“ beruht (Abb. 1) (Luhmann 2002, S. 171).

Abb. 1 illustriert die Wirkung des Containments. Wenn die elementaren Artefakte des Containments gröber granular sind als die entkoppelten Artefakte, werden direkte $\mathrm{m}: \mathrm{n}$-Zuordnungen (linke Seite) durch indirekte m:1- bzw. 1:nZuordnungen ersetzt. ${ }^{1}$ Diese repräsentieren gesamthaft nahezu die gleiche Semantik der direkten m:n-Zuordnung, verhindern aber gleichzeitig, dass jede Änderung in System A oder System B immer in das jeweils andere System propagiert werden muss.

Mit der Herleitung des ContainmentArtefakts beschreibt die Systemtheorie auf einer generischen Ebene den Nutzen und die Wirkung einer entkoppelnden Zuordnung. Durch das Containment können sowohl Artefakte des gleichen Typs wie auch Artefakte unterschiedlicher Typen entkoppelt werden. Das Containment kann sowohl real als auch virtuell umgesetzt werden. Im Folgenden sollen für diesen generischen Zuordnungsmechanismus Entsprechungen in der Informatik und der Ökonomie gefunden werden. Dabei werden ausschließlich solche Beispiele dargestellt, die Artefakte unterschiedlicher Typen entkoppeln und die ausschließlich der Zuordnung dienen, d. h. nicht Teil der zu entkoppelnden Strukturen sind.

\subsection{Entkoppelnde Zuordnungs- mechanismen in der Informatik}

Die offenkundigste Entsprechung zwischen der in Abschnitt 1 skizzierten Forderung und der in Abschnitt 3.1 generisch beschriebenen Umsetzung einer virtuellen, entkoppelnden Zuordnung ist die ANSI/ X3/SPARC-Norm 1975 für die Architektur von Datenbanksystemen. Diese als

\footnotetext{
1 Die Auflösung der direkten m:n-Zuordnung in $\mathrm{m}: 1-$ und 1:n-Zuordnungen stellt eine spezielle, stark vergröbernde Lösung dar. Eine allgemeine Lösung für die Vergröberung ist die Auflösung der direkten $\mathrm{m}: \mathrm{n}$-Zuordnung in $\mathrm{m}: \mathrm{x}$ - und $\mathrm{y}$ : $n$-Zuordnungen mit $m>x$ und $y<n$. Die folgenden Darstellungen illustrieren zur Vereinfachung die spezielle Lösung; Die Aussagen sind jedoch auch für die allgemeine Lösung gültig.
}

"Dreiebenenarchitektur" bekannte Vorschrift definiert, dass eine konzeptuelle Datenbank-Architekturebene allein dazu dient, Nutzungssichten auf Daten (externe Ebene) und die interne Implementierung der Daten (interne Ebene) voneinander zu entkoppeln. Anstelle einer direkten Zuordnung von implementierten Datenstrukturen (z. B. Records, Indizes) zu Nutzungssichten (z. B. unterschiedliche Strukturierungen von Kennzahlen in verschiedenen Unternehmensbereichen) wird in Form des konzeptuellen Datenmodells eine virtuelle (da weder unmittelbar implementierte noch unmittelbar genutzte) Zwischenebene eingeführt, auf die implementierte Datenstrukturen wie auch Nutzungssichten referenzieren. Der Nutzen dieser zusätzlichen Datenbank-Architekturschicht besteht darin, dass Änderungen der Datennutzung nicht immer auf die Datenspeicherung durchschlagen („logische Datenunabhängigkeit“") und dass Änderungen der Datenspeicherung nicht immer auf die Datennutzung durchschlagen („physische Datenunabhängigkeit") (Date 2000). Dieser Nutzen ist so groß, dass der Aufwand für die Abbildung und Aktualisierung zusätzlicher Artefakte gerechtfertigt ist.

In Unternehmen verändern sich fachliche Anforderungen und IT-Systeme durchaus unabhängig voneinander (z. B. Änderungen regulatorischer Rahmenbedingungen, Release-Änderungen bei eingesetzter Standardsoftware), so dass IT/ Business Alignment immer wieder hergestellt werden muss. Genauso treten in Datenbanken Änderungen der Implementierung von Datenstrukturen und Änderungen der Datennutzung unabhängig voneinander auf (z. B. Performance-Optimierungen auf der internen Ebene, neue Auswertungen auf der externen Ebene). Das konzeptuelle Datenmodell ist implementierungsneutral und integriert alle denkbaren Auswertungen, so dass jede Implementierung und jede Auswertung auf diese virtuelle Zuordnungsarchitektur abgebildet werden kann. Als Konsequenz muss auch die Zuordnungsarchitektur für IT/Business Alignment implementierungsneutral sein und möglichst viele fachliche Anforderungsvarianten abbilden können, um entkoppelnd zu wirken und die Pendants logischer und physischer Datenunabhängigkeit garantieren zu können.

Andere IT-Zuordnungsmechanismen wie z. B. das Data Warehouse (Devlin
1997; Inmon 1996) oder Enterprise Application Integration (Kaib 2002; Linthicum 2000) passen weniger gut zu den Anforderungen in Abschnitt 1: Durch diese Konzepte werden nicht autonome Architekturschichten mit möglicherweise unkoordinierten Änderungen voneinander entkoppelt, sondern es werden Artefakte des gleichen Typs (z. B. Softwarekomponenten) und damit der gleichen Architekturschicht miteinander verknüpft.

Zusammengefasst sind die wichtigsten Eigenschaften des ANSI/X3/SPARC-Beispiels:

- Es wird eine Architekturebene „Zwischen" Datennutzung und Datenspeicherung eingeführt.

- Diese Architekturebene dient primär zur „Übersetzung“ der verknüpften Strukturen und gehört weder zur Datennutzung noch zur Datenspeicherung.

- Die zusätzliche Architekturebene entkoppelt Artefakte verschiedener Typen.

- Direkte m:n-Zuordnungen werden zu indirekten m:1- bzw. 1:n-Zuordnungen aufgelöst.

- Die Artefakte auf den entkoppelten Ebenen können unabhängig voneinander verändert werden, ohne dass in jedem Fall die jeweils andere Ebene betroffen sein muss.

\subsection{Entkoppelnde Zuordnungs- mechanismen in der Ökonomie}

Aus der Perspektive der Ökonomie ist der Markt das Paradebeispiel eines Zuordnungsmechanismus - nämlich der Zuordnung von Angebot und Nachfrage nach einem ökonomischen Gut. Es wird nicht jedem Nachfrager überlassen, einen individuellen Anbieter zu finden und mit ihm eine direkte Punkt-zu-PunktZuordnung zu definieren, beispielsweise für Leistungsdefinitionen oder Preisverhandlungen. Bei einer größeren Menge an Anbietern und Nachfragern ist es effizienter, einen Markt als Zuordnungsarchitektur zwischenzuschalten (grundlegend Ricardo 1830; Smith 1776). Neben klassischen Marktfunktionen wie Preisbildung, Markträumung, Allokation und Effizienzsteigerung sind im hier betrachteten Fall vor allem die flexiblen Anpassungsfunktionen des Marktes für sich ändernde Rahmenbedingungen interessant (Fritsch et al. 2005; Herdzina 2005; Kantzenbach 1967; Kerber 2007). Auf 
beiden Seiten des Marktes (Anbieter- und Nachfragerseite) können (in bestimmten Grenzen) Veränderungen vorgenommen werden, ohne dass die jeweils andere Seite von diesen Änderungen betroffen sein muss. Der Markt „puffert" solche Veränderungen. So ist es im Normalfall unerheblich, wenn sich einzelne Anbieter vom Markt zurückziehen oder einzelne neue Anbieter hinzukommen. Die Nachfrager können die Produkte nach wie vor über den Markt beziehen. Analog kann sich auch die Nachfragerstruktur verändern, ohne dass die Anbieter darauf reagieren müssten. Im klassischen Sinne ist der Markt(-platz) der reale Ort des Warentauschs. Für die heute jedoch vielfach auch virtuellen Märkte gelten diese Entkopplungsmechanismen oft noch stärker, da sie häufig eine größere Anzahl von Anbietern und Nachfragern bündeln (Kollmann 2001; Weiber u. Kollmann 1998). Natürlich ist diese Darstellung insofern vereinfacht, als dass sie den für den Markt wesentlichen Mechanismus der Preisbildung und zunächst auch die Standardisierung des Guts außer acht lässt.

Werden die obigen Überlegungen um die Standardisierung des am Markt gehandelten Guts erweitert, werden die Ähnlichkeiten mit dem Entkopplungskonzept der Systemtheorie und seiner Realisierung in der Informatik noch deutlicher. Standards können für jegliche Art von Artefakten eine Zuordnungsarchitektur begründen, indem sie definieren, wie standardkonforme Artefakte unterschiedlichen Typs zueinander passen. Eine Vielzahl von Beispielen für die entkoppelnde Wirkung von Standards lassen sich etwa für den Bereich der Informationssysteme (Buxmann u. König 1998; Buxmann et al. 1999; Meffert 1993) oder auch für die Prozessgestaltung im Business-to-Business-Bereich finden (Dietrich 2008). Dem Nutzen der Standardisierung, standardkonforme Artefakte beliebig gegeneinander austauschen zu können, stehen Standardisierungskosten gegenüber. Dazu zählen die Kosten für die Definition des Standards, die standardkonforme Erstellung von Artefakten oder die Durchsetzung von Standards (Buxmann u. König 1998; Shapiro 2001). Je größer jedoch der Nutzerkreis eines Standards ist, um so eher werden diese Kosten durch die Realisierung von Netzeffekten überkompensiert (Katz u. Shapiro 1994). Bleiben die Standards jedoch sehr generisch und wei-
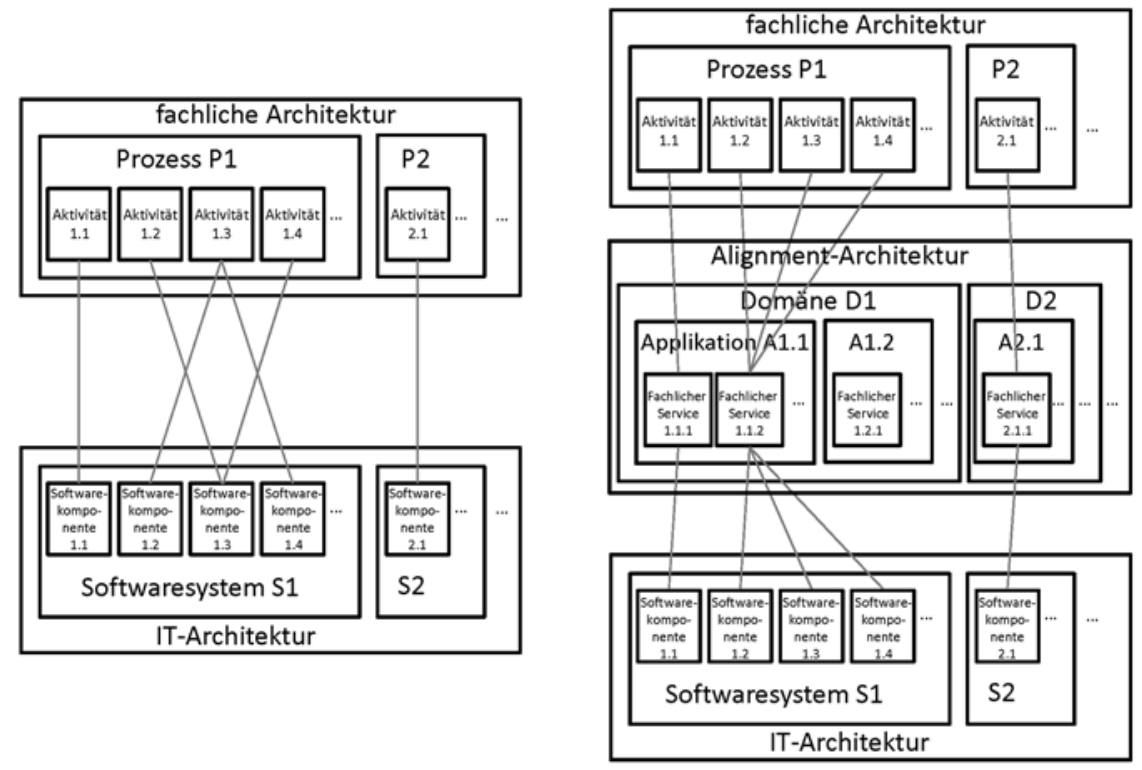

Abb. 2 Alignment-Architektur für die entkoppelnde Zuordnung fachlicher und IT-Artefakte

sen eine geringe Spezifität auf, so ist der verbleibende Definitionsaufwand für die Artefaktgestaltung groß und der unmittelbare Nutzen des Standards geringer.

Zusammengefasst sind die wichtigsten Eigenschaften des Markt- und Standardisierungsbeispiels:

- Es wird eine Architekturebene „zwischen" Angebot und Nachfrage eingeführt.

- Diese Zwischenebene kann (muss aber nicht) virtuell sein.

- Durch die Zwischenebene werden unterschiedliche Artefakttypen entkoppelt.

- Direkte m:n-Zuordnungen werden zu indirekten m:1- bzw. 1:n-Zuordnungen aufgelöst.

- Die Artefakte auf den entkoppelten Ebenen können unabhängig voneinander verändert werden, ohne dass in jedem Fall die jeweils andere Ebene betroffen sein muss.

- Die Einführung der Zwischenebene verursacht zusätzlichen Aufwand.

- Der Wertbeitrag der Zwischenebene steigt mit der Anzahl darüber angebundener Artefakte der angrenzenden Ebenen (Netzeffekte).

- Mit zunehmender Spezifität der Artefakte der Zwischenebene sinkt der Beitrag zur Entkopplung durch diese Ebene.

\section{Ein entkoppelnder Zuordnungsmechanismus für das IT/Business Alignment}

Ausgehend von den Anforderungen in Abschnitt 1 und in Analogie zu den Beispielen in Abschnitt 3 wird im Folgenden ein entkoppelnder Zuordnungsmechanismus - die Alignment-Architektur - abgeleitet, welcher eine flexible Integration von fachlichen und IT-Strukturen (IT/Business Alignment) unterstützt. Dazu werden zuerst die Anforderungen an den Zuordnungsmechanismus sowie an dessen Artefakttypen - die AlignmentArtefakttypen - definiert. Anschließend werden die Artefakttypen selbst spezifiziert.

\subsection{Anforderungen an die Alignment- Architektur und deren Artefakttypen}

Das Ziel besteht darin, fachliche Architekturen und IT-Architekturen durch Einführung eines Zuordnungsmechanismus so zu entkoppeln, dass Änderungen auf der Fachseite nicht zwangsläufig Änderungen in der IT nach sich ziehen und umgekehrt. Das konzeptionelle (Struktur-)Modell eines solchen Zuordnungsmechanismus wird als Alignment-Architektur bezeichnet und steht im Folgenden im Vordergrund der Betrachtungen. Daneben sind natürlich auch das implizierte Verhalten der Alignment-Architektur sowie zusätzliche, explizite Verhaltensas- 


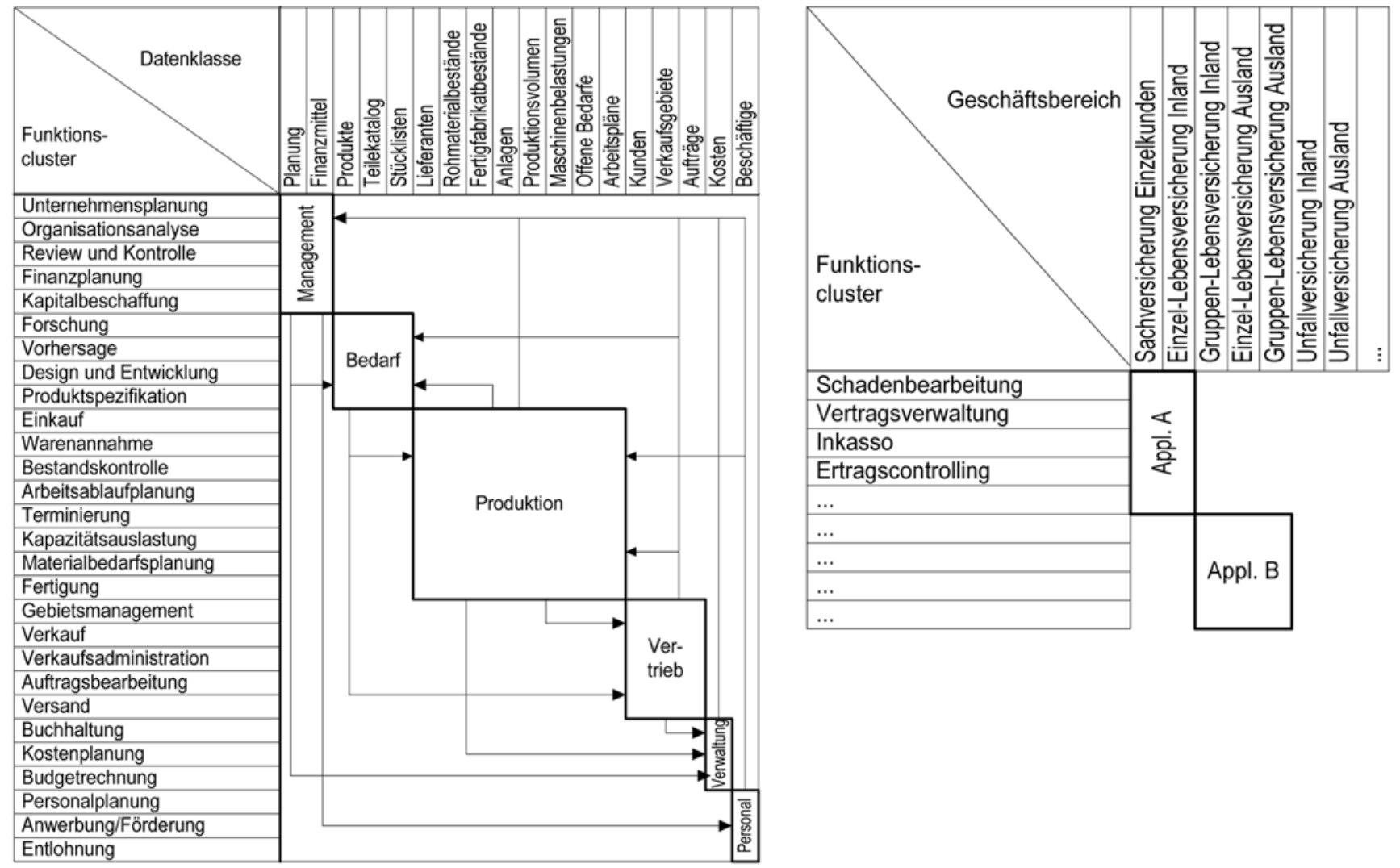

Abb. 3 BSP-Matrix und STP-Matrix

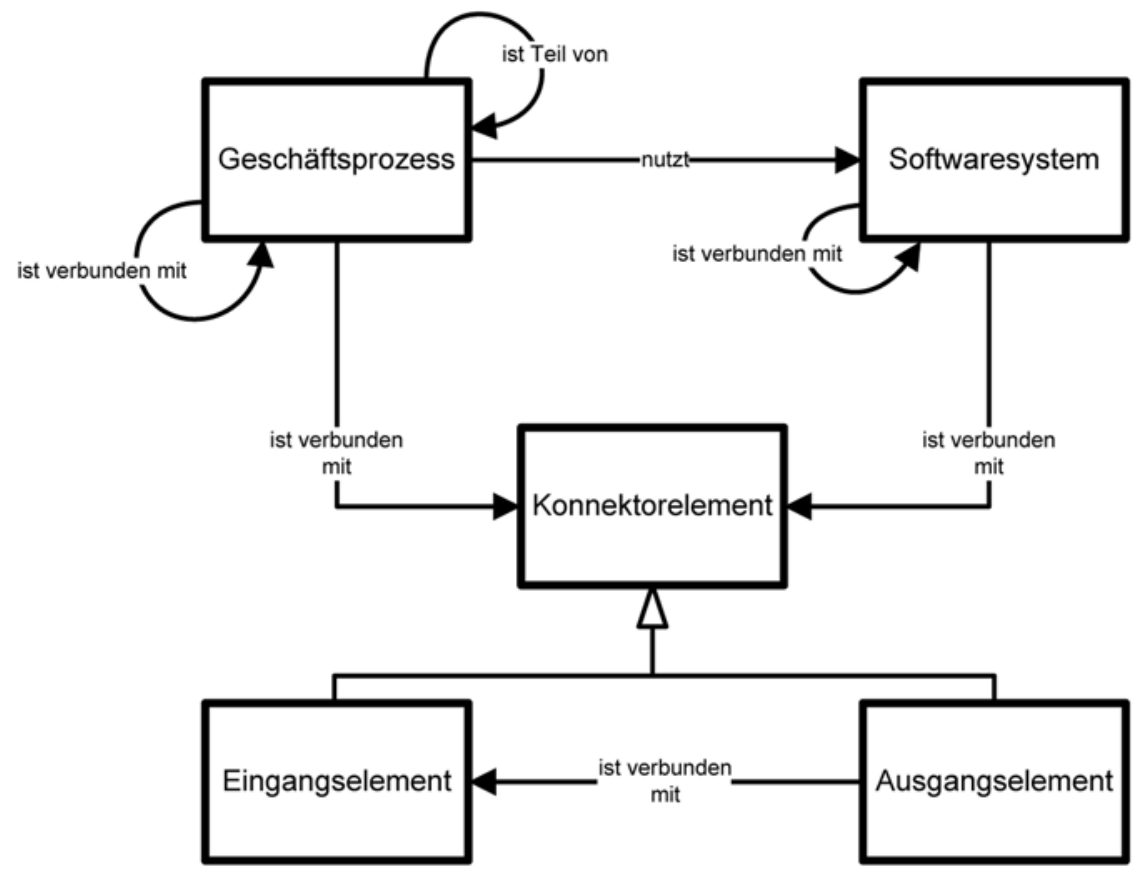

Abb. 4 Metamodell des Modellierungsansatzes

pekte des Zuordnungsmechanismus zu beschreiben. Diese Aspekte bilden den Gegenstand weiterführender Arbeiten zur Alignment-Architektur.
Zur strukturellen Beschreibung der Alignment-Architektur sind Artefakttypen zu spezifizieren, welche die direkten Beziehungen zwischen den Artefakten der fachlichen und IT-Architekturen durch
1. m:1-Beziehungen zwischen den Artefakten der fachlichen Architekturen und den Artefakten der AlignmentArchitektur sowie

2. 1:n-Beziehungen zwischen den Artefakten der Alignment-Architektur und den Artefakten der IT-Architekturen auflösen. Durch die m:1- bzw. 1:nAbbildung muss ein ausreichend großes Komplexitätsgefälle zwischen der Alignment-Architekturebene und den angrenzenden Ebenen erzeugt werden. Nur auf diese Weise kann eine Interdependenzunterbrechung zwischen fachlichen und IT-Architekturen erreicht werden. Ähnlich wie es innerhalb der fachlichen und IT-Architekturen Aggregationsebenen zur Vereinfachung gibt, müssen auch innerhalb der Alignment-Architektur Aggregationsebenen vorgesehen werden (Stünzer 1996, S. 73).

Analog zu den Beispielen in Abschnitt 3 dienen die Alignment-Architektur und deren Artefakte primär zur „Übersetzung“ der verknüpften Strukturen und sind weder Teil fachlicher Architekturen noch Teil der IT-Architekturen. 


\subsection{Artefakttypen der Alignment- Architektur}

Ausgehend von den Anforderungen des vorhergehenden Abschnitts 4.1 schlagen wir die Artefakttypen fachlicher Service, Applikation und Domäne für die Alignment-Architektur vor. Die Artefakttypen stehen in einer hierarchischen Beziehung zueinander (Abb. 2). Diese Beziehung muss nicht streng hierarchisch sein und kann somit auch eine Polyhierarchie darstellen.

Fachliche Services repräsentieren betriebswirtschaftlich abgeschlossene Funktionsbündel, die aufgrund der gemeinsamen Unterstützung von Geschäftsprozessen, der gemeinsamen Bewirtschaftung von Informationsobjekten oder gemeinsamer Wiederverwendung zusammenhängen (Schelp u. Winter 2008) und die durch Softwarekomponenten implementiert werden. Da es sich um Funktionsbündel handelt, werden sie durch eine oder mehrere Softwarekomponenten implementiert (1:n-Beziehung) und können in einem oder mehreren Geschäftsprozessen genutzt werden (m:1Beziehung). Applikationen sind Gruppierungen fachlicher Services, die zusammen bestimmte Geschäftsprozesse unterstützen, zusammen bestimmte Informationsobjekte bewirtschaften oder zusammen wiederverwendet werden (Winter 2008). Domänen sind Gruppierungen von Applikationen (und damit indirekt von fachlichen Services), welche aufgrund ihrer Nutzung in zusammenhängenden Geschäftsprozessen, ihrer Unterstützung durch zusammenhängende Softwarekomponenten oder ihrer Nutzung zusammenhängender Informationsobjekte einen Integrationsbereich bilden (Hagen 2003).

In Analogie zu Abb. 1 zeigt Abb. 2 die Alignment-Architektur zur indirekten, $\mathrm{m}$ : n-fähigen Zuordnung fachlicher und ITArtefakte. Wie in Systemtheorie, Informatik und Ökonomie wird eine neue Architekturebene „Zwischen“ fachlichen Architekturen und IT-Architekturen eingeführt. Die neue Architekturebene dient allein der „Übersetzung“ der verknüpften Strukturen und ist weder Teil fachlicher Architekturen noch von IT-Architekturen. Sie entkoppelt Artefakte verschiedener Typen. Direkte m:n-Zuordnungen werden zu m:1- bzw. 1:n-Zuordnungen aufgelöst. Die Artefakte auf den entkoppelten Ebenen können unabhängig voneinander verändert werden, ohne dass in jedem Fall die jeweils andere Ebene betroffen sein muss.
Da mit zunehmender Spezifität der Artefakte der Alignment-Architektur erwartet werden muss, dass der Wertbeitrag der zusätzlichen Architekturebene sinkt, sollten deren elementare Artefakte aggregierter als die entkoppelten Artefakte sein.

\section{Konstruktionsvorschriften für Artefakte der Alignment-Architektur}

Um die Alignment-Architektur zu entwickeln, sind geeignete fachliche Services, Applikationen und Domänen zu identifizieren; Außerdem sind sinnvolle Zuordnungen von fachlichen Services zu Applikationen und von Applikationen zu Domänen zu finden. Für den Entwurf fachlicher Services wurden erste Ansätze (Heutschi u. Legner 2007; Schelp u. Winter 2007) entwickelt. Dabei werden ausgehend von den verfolgten Zielen abstrakte Konstruktionsvorschriften abgeleitet, auf Basis derer fachliche Services eher datenorientiert, eher prozessorientiert oder eher wiederverwendungsorientiert "geschnitten“ werden (Schelp u. Winter 2008).

Im Gegensatz zum Entwurf fachlicher Services bestehen für den Applikationsentwurf langjährige Erfahrungen (IMG 1999; Winter 2003).

Basierend auf Vorläufern in den 1960erJahren hat die Business-Systems-Planning-Methode von IBM (BSP, IBM 1984) in den 1980er-Jahren weltweite Verbreitung gefunden. Das Ziel von BSP ist es, datennutzungsorientiert IT-Funktionalitäten zu gruppieren um somit Applikationen zu identifizieren. Eine alternative Methode des Applikationsentwurfs wird in der Systems-and-Technology-Planning-Methode von IMG (STP, IMG 1999) vorgeschlagen. IT-Funktionalitäten werden in STP nicht aufgrund „ähnlicher“ Datennutzung, sondern aufgrund „ähnlicher" Verantwortlichkeiten zu Applikationen gruppiert (Abb. 3).

Der systematische Domänenentwurf wurde in der Wirtschaftsinformatik bislang nur wenig und vorwiegend in Praktikerbeiträgen behandelt (Bath u. Herr 2004; Hagen 2003). Ähnlich dem Entwurf fachlicher Services werden auf Basis abstrakter Regeln (z. B. „Minimiere die Abhängigkeiten der Elemente unterschiedlicher Domänen und maximiere die Abhängigkeiten innerhalb einer Domäne") in einem Top-down-Ansatz Domänen definiert.

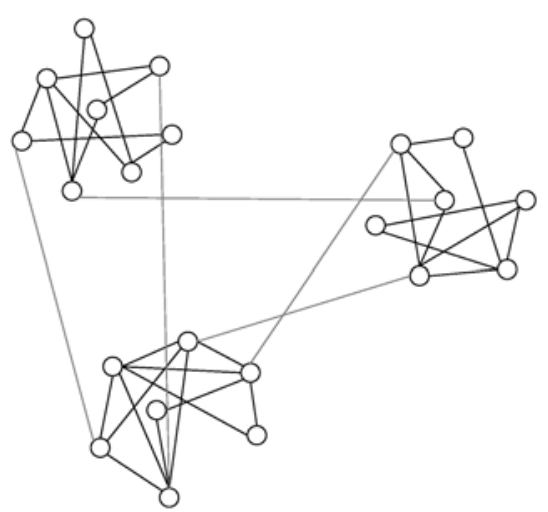

Abb. 5 Soziales System mit einer Community-Struktur (Girvan u. Newman 2002, S. 7822)

Bestehende Konstruktionsvorschriften für fachliche Services und Domänen sind fast immer durch ein Top-down-Vorgehen gekennzeichnet. Hingegen verfolgt BSP zwar einen Bottom-up-Ansatz, adressiert jedoch durch die Analyse ausschließlich technischer Artefakte (IT-Funktionalitäten und Datenstrukturen) kein IT/ Business Alignment. STP verfolgt ebenfalls einen Bottom-up-Ansatz und adressiert teilweise technische, teilweise fachliche Artefakte (IT-Funktionalitäten und Organisationseinheiten), berücksichtigt aber weder Datenkopplung noch Wiederverwendung bei der Applikationsbildung. Würden bei der Applikationsbildung die Abhängigkeiten zwischen fachlichen Strukturen (z. B. Geschäftsprozessen) und technischen Strukturen (z. B. Softwaresystemen) analysiert und beschränkte sich die Analyse nicht nur auf eine Kopplungsdimension, so ließen sich mit diesem Bottom-up-Ansatz geeignete, grob granulare Alignment-Artefakte finden.

In einer empirischen Analyse zeigen Schelp u. Winter (2008), dass die Entwurfsprinzipien für fachliche Services denen von Applikationen entsprechen. Dieser Befund legt die Vermutung nahe, dass die Grenze zwischen fachlichen Services, Applikationen und Domänen fließend ist und nur durch den jeweiligen Aggregationsgrad, nicht aber durch unterschiedliche Entwurfsprinzipien definiert ist. In einer mehrstufigen Aggregationshierarchie stellen Domänen die gröbste Ebene und fachliche Services die feinste Ebene dar. Dazwischen findet je nach Komplexität der zu verknüpfenden Architekturen der Übergang von Domänen zu Applikationen und von Applikationen zu fachlichen Services früher oder später statt. 


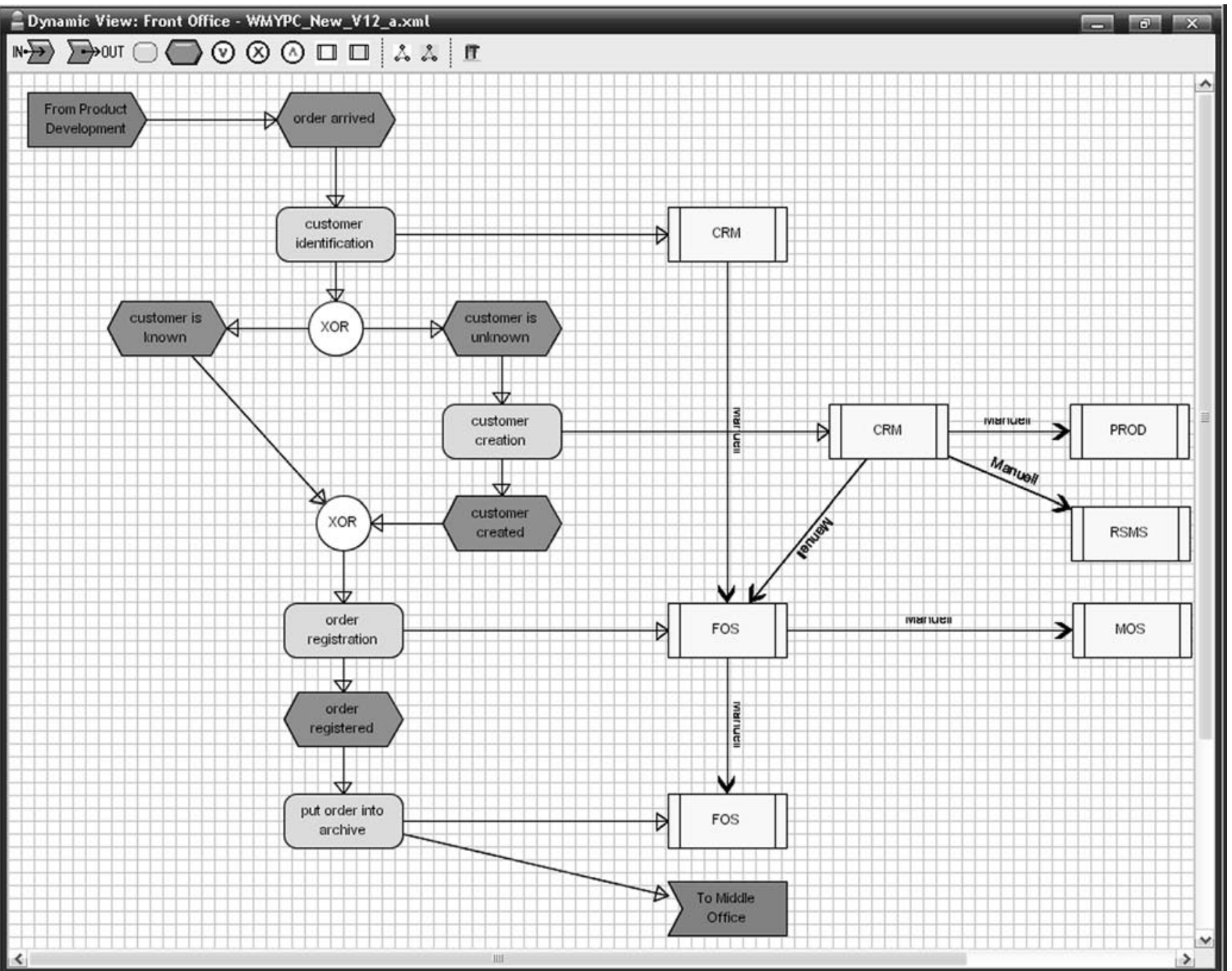

Abb. 6 Modellierung im Prototyp (Ausschnitt des Modells)

Für die Konstruktion der AlignmentArtefakte schlagen wir darum eine Bottom-up getriebene Analyse der Beziehungen zwischen fachlichen Architekturen und IT-Architekturen vor. Das Ergebnis dieser Analyse sind, je nach Detaillierungsebene der analysierten Beziehungen, fachliche Services, Applikationen oder Domänen.

Im folgenden Abschnitt stellen wir das Bottom-up-Vorgehen für die Identifikation von Alignment-Artefakten am Beispiel der Domänenbildung im Detail vor.

\section{Ein exploratives Bottom-up- Vorgehen zur Identifikation von Alignment-Artefakten am Beispiel der Domänenbildung}

Im Folgenden wird ein formaler Clustering-Algorithmus angewendet, um
Domänen auf Basis der Organisationsund Softwarearchitektur zu identifizieren. Um einen solchen Algorithmus anwenden zu können, bedarf es eines entsprechenden Modells der Organisations- und Softwarearchitektur. Zuerst wird das zugrunde liegende Metamodell abgeleitet. Darauf folgen die Einführung des verwendeten Clustering-Algorithmus sowie die Darstellung der prototypischen Implementierung.

\subsection{Ableitung des Metamodells}

In unserem Modellansatz liegt der Schwerpunkt darauf, die Verknüpfungen zwischen der fachlichen Organisationssicht sowie der IT-Sicht abbilden zu können. Für das entsprechende Metamodell werden stark vereinfachend die folgenden Elemente berücksichtigt:

- Geschäftsprozesse auf hohem Aggregationsniveau,
- Softwaresysteme auf hohem Aggregationsniveau,

- Verknüpfungen, die die Nutzung von Softwaresystemen entlang eines Geschäftsprozesses widerspiegeln

- sowie die Verknüpfungen zwischen Softwaresystemen entlang eines Geschäftsprozesses.

Um in beliebigen Detaillierungsebenen konsistent modellieren zu können, wurden Eingangs- und Ausgangselemente als Konnektoren zwischen verschiedenen Detailierungsebenen hinzugefügt (Abb. 4). ${ }^{2}$ Für die spätere Anwendung des Clustering-Algorithmus ist es wichtig, die zu analysierenden Bereiche bei der Instanziierung des Metamodells konsistent und ausgerichtet am angestrebten Detaillie-

\footnotetext{
Das Konnektorelement und seine

Spezialisierungen haben also vor allem eine modellierungstechnische Funktion, um

sicherzustellen, dass sich aus dem Modell ein zusammenhängender Graph über verschiedene Detaillierungsstufen erzeugen lässt.
} 


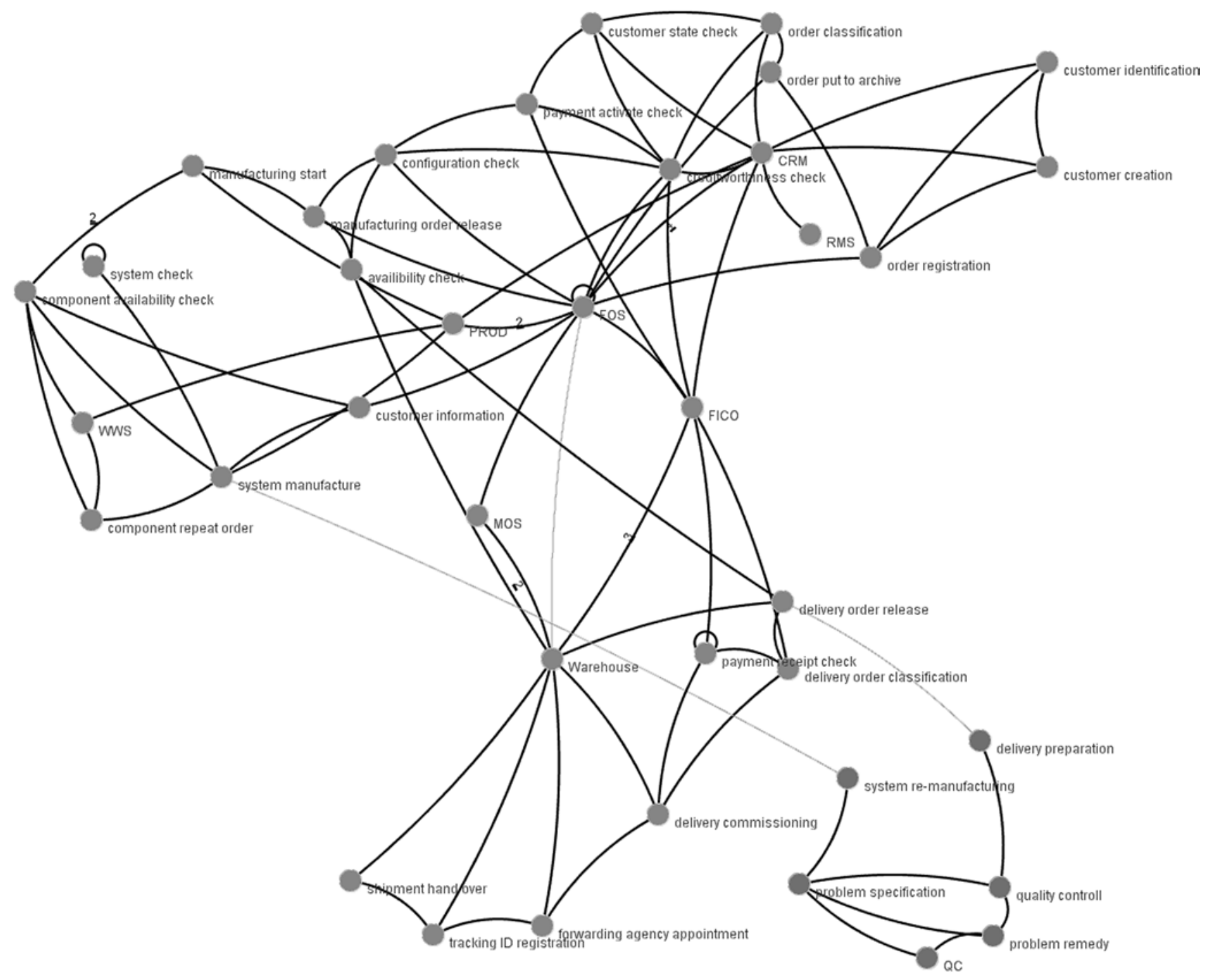

Abb. 7 In einen Graphen transformiertes Modell

rungsgrad vollständig zu modellieren. Insbesondere dürfen keine „Lücken“ im Modell und dadurch unverbundene Modellteile auftreten, da diese das Ergebnis des Clusterings unbrauchbar machen würden.

\subsection{Formalisierung der Domänenbildung}

Instanzen des Alignment-ArchitekturMetamodells können durch Transformation in einen Graphen überführt werden. Im Folgenden wird eine kurze Einführung in die Graphentheorie sowie in die Algorithmen zum Clustering von Graphen gegeben. Anschließend wird die Implementierung dieser Algorithmen für das Clustering der modellierten Artefakte der fachlichen Architektur und der ITArchitektur vorgestellt. Da diese Artefakte auf einer aggregierten Ebene betrachtet werden, bestimmen die so entstehenden
Cluster die Alignment-Architektur auf der Detaillierungsebene von Domänen.

Ein Graph besteht aus einer Menge von Knoten (vertices) $V$ und einer Menge von Kanten (edges) E. Alle strukturell wesentlichen Elemente unseres Modells (Geschäftsprozesse, Softwaresysteme) lassen sich als Knoten und die Verknüpfungen (Nutzung von Softwaresystemen in Geschäftsprozessen, Steuerungsflüsse, Softwaresystemschnittstellen) zwischen diesen Elementen als Kanten abstrahieren.

Graphen, deren Kanten durch Zahlenwerte attributiert werden, heißen gewichtete Graphen. Kanten, die einen definierten Anfang und ein definiertes Ende haben, heißen gerichtete Kanten. Kanten, bei denen das nicht der Fall ist, heißen ungerichtete Kanten. Die Entfernung zwischen zwei Knoten ist definiert als der kürzeste Pfad zwischen diesen. Bei gewichteten Graphen wird oft auch von der gewichten Entfernung gesprochen (O’Madadhain et al. 2005, S. 6). Ein solcher Graph mit $n$ Knoten lässt sich nun als Nachbarschaftsmatrix $A$ der Form $n \times n$ mit den Elementen

$a_{i j}=\left\{\begin{array}{cc}1 & \text { wenn die Knoten } i \text { und } j \\ 0 & \text { verbunden sind }\end{array}\right.$

beschreiben. In einem gewichteten Graphen stellt $a_{i j}$ das Gewicht der Kante, welche die Knoten $i$ und $j$ verbindet, dar. Weiterhin lässt sich der Grad (degree) $k$ eines Knotens $i$ definieren als die Anzahl der Kanten, die mit diesem Knoten verbunden sind:

$k_{i}=\sum_{j} a_{i j}$

Bei der Transformation unserer Modelle in einen Graphen ist es zunächst unerheblich, 


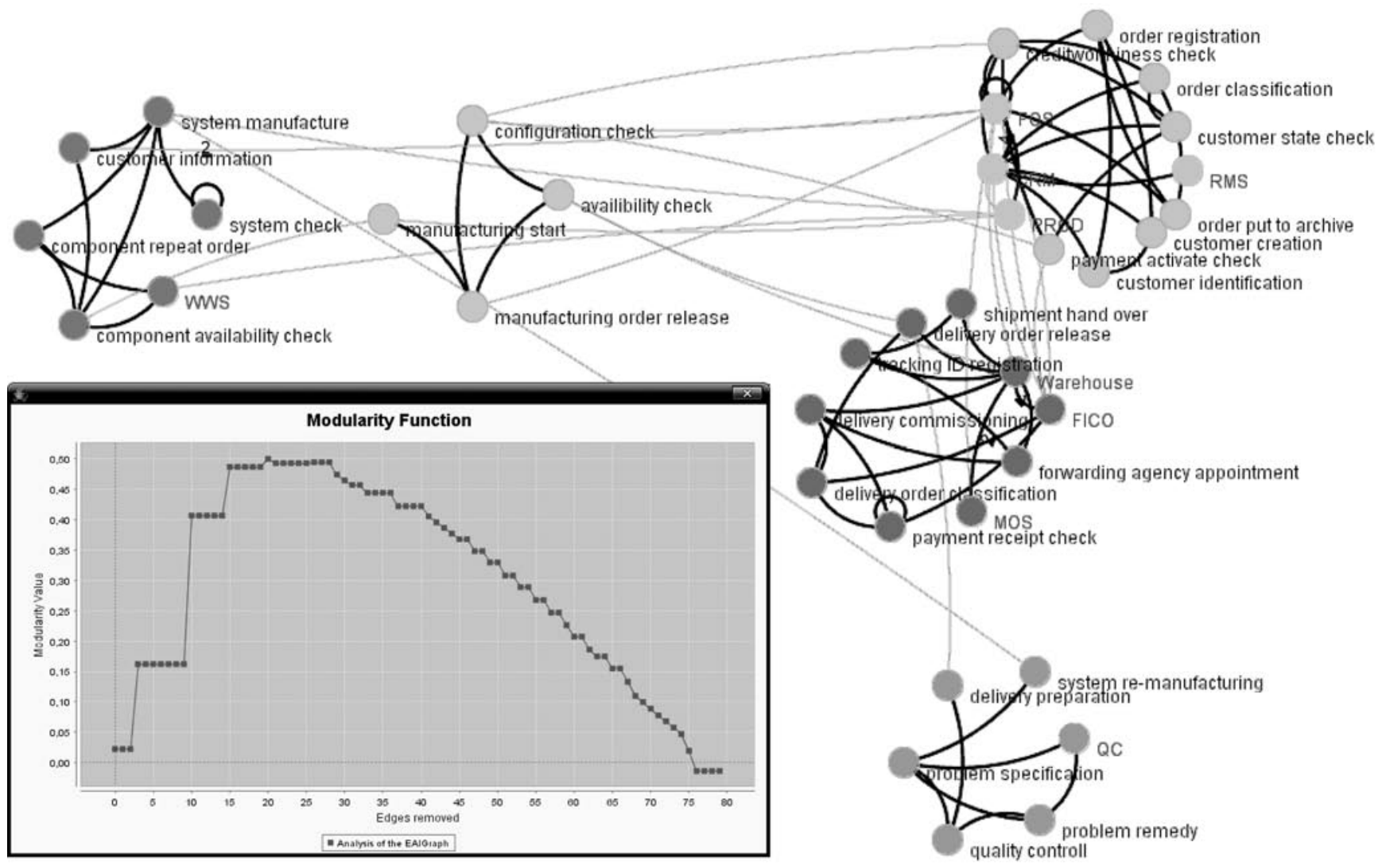

Abb. 8 In einen Graphen transformiertes Modell nach dem Entfernen von 20 Kanten

welchem Typ die Elemente entsprechen. Sie werden gleichrangig als Knoten abgebildet. Die Kanten werden als ungerichtete Kanten in den Graphen übernommen, da die Richtungsinformation hier nicht wesentlich für die strukturellen Zusammenhänge ist. Die Gewichte an den Kanten ergeben sich aus der Anzahl der Kanten, welche zwei Knoten miteinander verbinden. Ein Gewicht größer als eins kommt immer dann vor, wenn die Verbindung zweier Softwaresysteme in unterschiedlichen Prozessen genutzt werden.

Aus den Beispielen in Abschnitt 3 sowie den Anforderungen in Abschnitt 4.1 lassen sich als die wichtigsten Kriterien für die Domänenbildung die Minimierung der Abhängigkeiten zwischen verschiedenen Domänen und die Maximierung der Abhängigkeiten zwischen Elementen innerhalb einer Domäne (im Sinne einer Verschiebung solcher Elemente in eine Domäne) extrahieren. Bereits bei kleineren Ausschnitten einer fachlichen und einer IT-Architektur wird die Anwendung einer solchen Vorschrift ohne Werkzeugunterstützung schnell schwer überschaubar. Darum wurde versucht, Algorithmen zu finden und anzuwenden, die aus der Struktur eines Gra- phen die Zusammengehörigkeit verschiedener Elemente bewerten. Dabei wird von der Semantik der Modellelemente abstrahiert und ausschließlich die Modellstruktur ausgewertet. Entsprechend des Analyseansatzes sollten alle Elemente mit einer hohen Zusammengehörigkeit eine Domäne bilden.

Solche Fragestellungen werden unter den Schlagworten Partitionierung bzw. Clustering von Graphen diskutiert. Ein Cluster ist dabei eine Menge von Elementen, die in der einen oder anderen Form ähnlich zueinander sind (O'Madadhain et al. 2005, S. 18). Im hier vorliegenden Fall ergibt sich Ähnlichkeit aus der Tatsache, dass verschiedene Elemente eine gemeinsame Untermenge von Nachbarn haben.

Girvan u. Newman (2002, S. 7821) haben einen Clustering-Algorithmus entwickelt, um Communitys in sozialen Systemen zu bestimmen. Ein solches System besteht dabei aus einer Menge von Personen (Knoten) die Beziehungen zueinander haben (Kanten). Die Beziehung kann beispielsweise durch das gegenseitige Kennen bestehen. Die Frage ist nun, welche Personen sich kennen und ob sich diese Personen und die Communitys zu denen sie gehören (Cluster) identifizieren lassen?
Abb. 5 stellt ein solches System dar, in welchem die Cluster (Communitys) hervorgehoben worden sind. Sowohl Girvan und Newman als auch wir haben bestehende Clustering-Algorithmen nach ihrer Leistungsfähigkeit analysiert.

In bestimmten Konstellationen (insbesondere bei Knoten am Rand eines Graphen, welche nur durch eine einzige Kante mit dem übrigen Graphen verbunden sind) liefern diese Algorithmen schlechte Ergebnisse. Elemente werden aus einem Cluster ausgeschlossen, obwohl sie eigentlich dazugehören sollten. Andere Algorithmen wie beispielsweise der Voltage-Clusterer (Wu u. Huberman 2004) wurden ebenfalls getestet, führten in diesem Kontext jedoch nicht zu brauchbaren Ergebnissen.

Die Grundidee des Algorithmus von Girvan und Newman ist es nicht, die zentralen Kanten eines Graphen zu finden. Vielmehr sollen Kanten identifiziert werden, welche am wenigsten zentral sind und somit am meisten $z$ wischen (between) den Communitys liegen. ${ }^{3}$ In der Vergan-

\footnotetext{
3 Im Folgenden wird angelehnt an den Originalbeitrag von Girvan u. Newman (2002) weiter von Betweenness gesprochen als einem Grad, wie sehr ein Element zwischen den anderen liegt. Es gibt $u$. E. keine prägnante deutsche Übersetzung.
} 


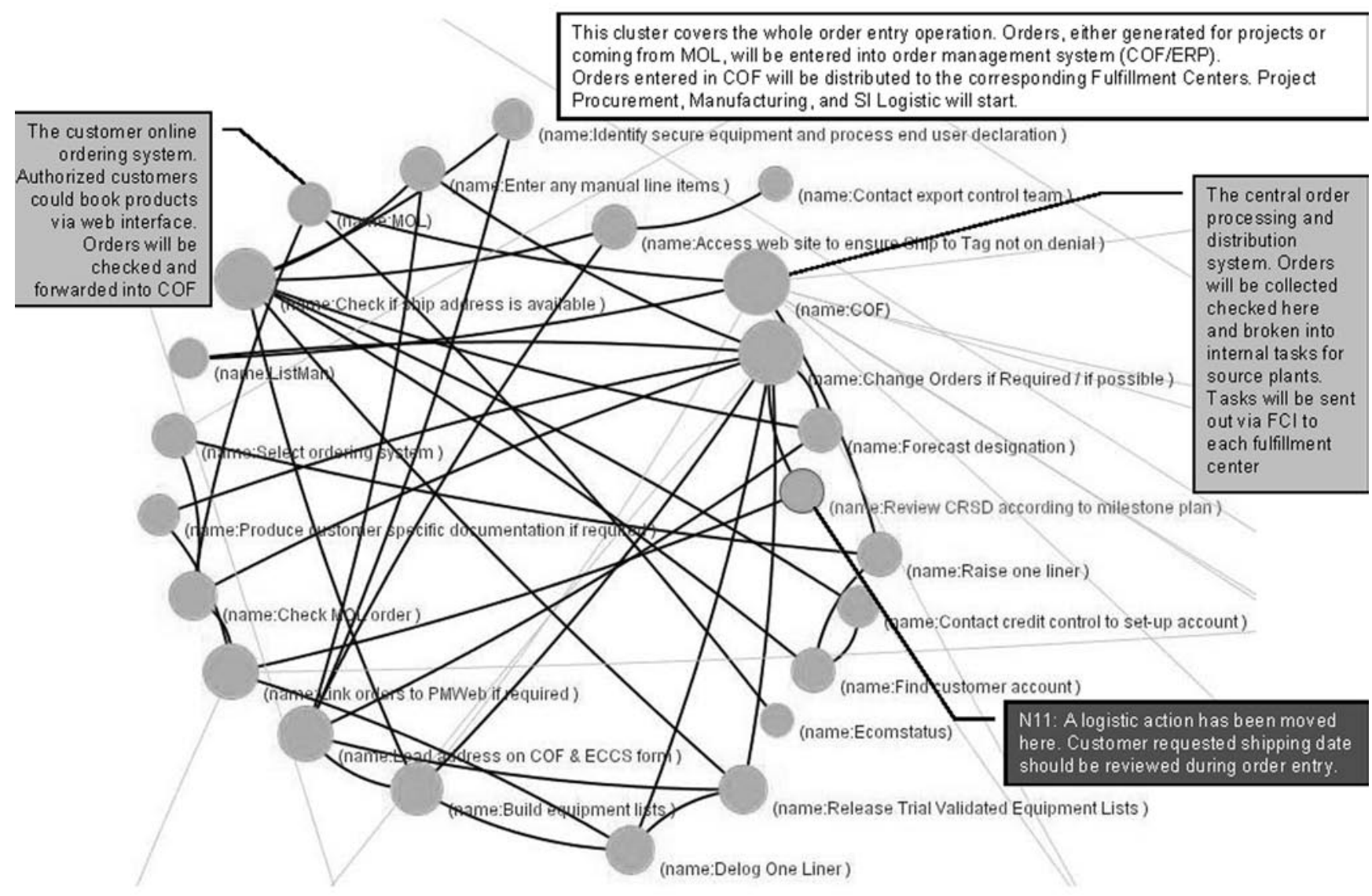

Abb. 9 Domäne Auftragsmanagement im Rollout eines Funksystems

genheit wurde die Knoten-Betweenness als ein Maß des Einflusses eines Knotens auf einen Graphen untersucht. Zuerst von Freeman (1977) vorgeschlagen, wird die Betweenness-Centrality eines Knotens $i$ definiert als die Anzahl der kürzesten Pfade zwischen Paaren anderer Knoten, die durch $i$ verlaufen. Girvan und Newman haben nun Freemans BetweennessCentrality verallgemeinert und definieren die Kanten-Betweenness einer Kante als die Anzahl der kürzesten Pfade zwischen Paaren von Knoten, die über diese Kante laufen. Wenn in einem System Communitys bestehen, dann sind diese nur über wenige Kanten miteinander verbunden. Das heißt, dass die kürzesten Pfade zwischen diesen Communitys über diese wenigen Kanten laufen. Darum werden diese Kanten eine hohe Kanten-Betweenness haben. Durch das Entfernen dieser Kanten können die Communitys separiert werden. Damit wird die zugrunde liegende Struktur aufgedeckt.

Dieser so genannte Edge-Remover-Algorithmus für gewichtete Graphen ist wie folgt anzuwenden (Newman 2004, S. 4):
1. Berechnung der Betweenness für alle Kanten in einem Graphen.

2. Division der Betweenness durch das Gewicht der jeweiligen Kante.

3. Entfernung der Kante mit der höchsten resultierenden Betweenness.

4. Neuberechnung der resultierenden Betweenness für alle übrigen Kanten.

5. Wiederholung der Schritte 3 und 4, bis keine Kanten übrig bleiben.

Eine weitere Frage ist, wann eine gutes Clustering (eine gute Spezifität unserer Domänen) durch das Entfernen von Kanten erreicht ist bzw. wann andernfalls noch weitere Kanten entfernt werden sollten. Newman (2004, S. 7) definiert dazu die Modularität Q. Er berechnet den Anteil der Kanten eines Graphen, welche sich innerhalb der Communitys befinden, wie folgt:

$$
\frac{\sum_{i j} a_{i j} \delta\left(c_{i}, c_{j}\right)}{\sum_{i j} a_{i j}}=\frac{1}{2 m} \sum_{i j} a_{i j} \delta\left(c_{i}, c_{j}\right)
$$

Dabei ist $c_{i}$ die Community, welcher der Knoten $i$ angehört. Die Funktion $\delta(u v)$ ist dann 1 , wenn $u=v$. Ansonsten ist sie 0 . Weiterhin ist $m=\frac{1}{2} \sum_{i j} a_{i j}$ die Anzahl der Kanten des Graphen. Wenn nun der $k_{i}$ für alle Knoten $i$ beibehalten wird, jedoch die Kanten zufällig im Graphen verteilt werden, dann ist die Wahrscheinlichkeit, dass zwischen den Knoten $i$ und $j$ eine Kante existiert $k_{i} k_{j} / 2 m$. Daraus ergibt sich die Modularität $Q$ als

$$
Q=\frac{1}{2 m} \sum_{i j}\left[a_{i j}-\frac{k_{i} k_{j}}{2 m}\right] \delta\left(c_{i}, c_{j}\right) .
$$

Daraus ergeben sich für $Q$ Werte zwischen 0 und 1. Ein Wert von 0 bedeutet, dass es nach einem Clustering keine Kanten mehr in einer Community gibt, die man dort gemäß Zufall erwarten würde. Reale Werte für $Q$, welche ein gutes Clustering vermuten lassen, liegen zwischen 0,3 und 0,7 .

\subsection{Prototypische Implementierung}

Der Modellierungs- und ClusteringAnsatz für den hier dargestellten Kontext wurde in einem Prototyp implementiert.

In Abb. $\mathbf{6}$ ist ein Ausschnitt des zugrunde liegenden Modells im Prototyp dargestellt. 
In diesem Fall wurden die Geschäftsprozesse in Form einer ereignisgesteuerten Prozesskette (EPK) modelliert. Zusätzlich wurden die Softwaresystemnutzung entlang der Geschäftsprozesse, die Schnittstellen zwischen den Softwaresystemen entlang des Prozessflusses sowie die Einund Ausgänge für die hierarchische Verfeinerung der Modelle abgebildet.

Die Leistung des eingesetzten Clustering-Algorithmus wurde an einer Reihe spezieller Testszenarien untersucht (Aier 2006). Bevor im folgenden Abschnitt die Anwendung des Domänen-Clusterings anhand einer realen Fallstudie diskutiert wird, soll zuerst die Funktionsweise anhand eines einfachen Testszenarios demonstriert werden.

Abb. 7 zeigt das zum Testszenario gehörige Modell, welches in einen Graphen transformiert wurde. ${ }^{4}$ Bei 20 entfernten Kanten erreicht die Modularitätsfunktion ihr Maximum. Nach der Anwendung des Clustering-Algorithmus und dem Entfernen von 20 Kanten (Abb. 8) ist der Graph in fünf Cluster aufgeteilt. Abb. 8 zeigt die entstandenen Cluster in einer gruppierten Ansicht sowie den Verlauf der zugehörigen Modularitätsfunktion über der Anzahl entfernter Kanten.

Das Ziel des hier vorgestellten Ansatzes ist es, eine Domänen-Architektur auf der Alignment-Ebene in einem Bottom-upAnsatz aus den bestehenden Softwareund Ablauforganisationsstrukturen abzuleiten. Dafür ist es wichtig, die zentralen Kopplungselemente zu identifizieren. Der angewendete Clustering-Algorithmus findet durch die Anwendung auf die bestehenden Architekturen die Kandidaten für die Domänen auf der Alignment-Ebene. Ein Cluster ist genau das Element, auf welches m Elemente der fachlichen Architektur und n Elemente der IT-Architektur abgebildet werden. Dabei werden möglichst viele Elemente auf ein Cluster abgebildet - ohne jedoch zu viel Spezifität zu verlieren.

\subsection{Fallstudie eines \\ Telekommunikationsausrüsters}

Um die Leistung des vorgeschlagenen Ansatzes zur Domänenbildung neben Experimenten an Testmodellen auch für reale Fälle zu evaluieren, wurde eine

\footnotetext{
4 Bei der Transformation des Modells in den Graphen wurden die Ereignisse und Operatoren eliminiert, da sie in einem ungerichteten Graphen keine Bedeutung für die Struktur haben.
}

umfangreiche Fallstudie durchgeführt. Im Folgenden werden zuerst das Unternehmen und die konkrete Situation beschrieben, um anschließend die Ergebnisse darzustellen und zu diskutieren.

\subsubsection{Unternehmen und Situation}

Die Studie wurde bei einem amerikanischen, weltweit tätigen Telekommunikationsausrüster durchgeführt. Das Unternehmen gehört zu den Fortune-100 und wies 2007 einen Jahresumsatz von 36,6 Milliarden US-Dollar aus. Der betrachtete Bereich produziert, konfiguriert und verkauft weltweit professionelle Funksysteme für Firmenkunden und Kunden der öffentlichen Verwaltung.

Ausgangspunkt für die Fallstudie war ein Projekt, in welchem die komplette Softwarelandschaft des betrachten Bereichs an den fachlichen Strukturen ausgerichtet werden sollte. Das Ziel des Projekts war es, die latent vorhandene Struktur der Gesamtheit von Softwaresystemen und Geschäftsprozessen in Form eines Domänenmodells zu identifizieren. Darauf aufbauend sollte für Domänen mit besonders hohem Handlungsbedarf eine Neugestaltung der IT-Architektur vorgenommen werden, um eine effiziente Entkopplung unabhängiger Artefakte zu ermöglichen und damit das IT/Business Alignment zu fördern. Der hier betrachtete Bereich umfasst ca. 100 Softwaresysteme und ca. 400 Teilprozesse nebst zugehöriger Ereignisse, Operatoren und Beziehungen im Prozessmodell.

Das betrachtete Geschäft reicht dabei inhaltlich von einer unstrukturierten Marktbeobachtung im Vorfeld der Teilnahme an Ausschreibungsverfahren über die Erstellung des Funksystems im zentralen Integrationszentrum, die Installation an den verteilten Standorten, den Betrieb des Systems über ca. 25 Jahre und den anschließenden Abbau und die Entsorgung des Systems. Dazu wurden zuerst mit Hilfe der Inventurmethode und mit Interviews die Geschäftsprozesse, die Softwaresystemlandschaft sowie die Verbindungen zwischen beiden aufgenommen und in einem Modell mit Hilfe des Prototypen dokumentiert (Frank et al. 2007, S. 152-159, 165-168).

\subsubsection{Ergebnisse}

Durch die Anwendung des ClusteringAlgorithmus auf das Modell wurden 44
Domänen identifiziert. In Abb. 9 ist eine dieser Domänen beispielhaft abgebildet. Den Domänen wurden in der folgenden manuellen Analyse Namen gegeben und es wurden 24 auffällige Eigenschaften der Domänen im Cluster-Annotation-Report dokumentiert. Dies waren beispielsweise Domänen ohne IT-Unterstützung. In anderen Fällen wurden Elemente einer anderen Domäne als erwartet zugeordnet. Dabei handelte es sich um sechs aus fachlicher Sicht Fehlgruppierungen, neun offensichtliche Verbesserungspotenziale und neun zu diskutierende Potenziale wie Änderungen des Geschäftsprozesses, Möglichkeiten der künftigen IT-Unterstützung etc.

Für die beteiligten Anspruchsgruppen ermöglichte die Diskussion der Clustering-Ergebnisse ein Verständnis der latent vorhandenen Strukturen. Die Diskussion der Cluster war eine gute Übung, um das Zusammenspiel von Softwaresystemen und Prozessen zu verstehen sowie Lücken in der IT-Unterstützung bestimmter Prozesse zu entdecken und zu verstehen.

\subsubsection{Diskussion der Fallstudie}

Die Domänenbildung wurde mit den betroffenen IT- bzw. Prozessverantwortlichen analysiert. Im Ergebnis konnte festgestellt werden, dass die entstandenen Cluster fachlich sinnvoll sind. Dennoch waren die Cluster für die Verantwortlichen zum Teil überraschend, da sie nicht immer die organisatorisch festgeschriebenen Gruppierungen und Kompetenzen widerspiegelten. Daraus lässt sich jedoch nicht schließen, dass die Organisationsstrukturen entsprechend der Clustering-Ergebnisse verändert werden sollten. Nach Frese (1993, S. 1021) sind diese Einheiten/Domänen „keine Blaupause zur Umstrukturierung von Großunternehmungen“, sondern sie sind bereichsüberlagernde (und somit virtuelle) Sekundärstrukturen mit einer Leitbildfunktion für die Prinzipien der Überschaubarkeit und Abgeschlossenheit. Sie liefern in unserem Kontext also grob granulare Alignment-Artefakte, welche die Geschäftsprozesse und die Softwaresysteme durch Verknüpfung integrieren. Diese Alignment-Artefakte sind geeignet, eine Interdependenzunterbrechung zwischen der fachlichen Architektur und der IT-Architektur herbeizuführen.

Die identifizierten Domänen stellen Artefakte der Alignment-Architek- 
tur dar, welche die Artefakte der angrenzenden Ebenen einander zuordnen. Zwischen den Domänen ist zu überprüfen, ob die für die Domänenbildung „gelöschten“ Kanten ebenfalls konsolidiert werden können, um dem Ziel der losen Kopplung in einer Soll-Architektur näher zu kommen. So kann eine Reduktion der durch den Algorithmus entfernten Kanten pro Cluster als Qualitätsindikator für die fachliche und die IT-Architektur gelten. Insbesondere die Softwaresysteme, die sehr viele Kanten zu Elementen anderer Domänen aufweisen (beispielsweise das Softwaresystem „COF“ in Bild 9), sollten auf einer detaillierteren Ebene in verschiedene Softwareservices aufgebrochen werden. Hierin liegen die größten Potenziale für eine weitere Entkopplung der Architekturebenen. Änderungen, die sich dann nur auf Teile des heutigen „COF-Systems“ beziehen, bleiben in ihrer Wirkung lokal begrenzt und können gezielt über die AlignmentArtefakte der fachlichen Architektur zugeordnet werden.

Einschränkend muss erwähnt werden, dass aussagefähige Ergebnisse des Clusterings immer einer sorgfältigen und vor allem konsistenten Modellierung bedürfen, da Modellierungsentscheidungen das Ergebnis des Clusterings stark beeinflussen. Gleichzeitig sind die strukturellen Zusammenhänge schon bei der in dieser Fallstudie vorliegenden Komplexität der fachlichen und der IT-Architektur nicht mehr intuitiv erkennbar, sodass die Anwendung des vorgestellten Algorithmus tatsächlich Nutzen für die Analyse der fachlichen und IT-Architekturen und die Gestaltung der Alignment-Architektur liefert.

\section{Diskussion: Konsequenzen und Ausblick}

Dieser Beitrag untersucht die Integration fachlicher Architekturen und IT-Architekturen als eines der Kernthemen der Wirtschaftsinformatik. Ausgehend vom generellen Entkopplungsansatz der Systemtheorie und von entsprechenden Beispielen aus der Informatik und der Ökonomie wird die Entkopplung von fachlichen Architekturen und IT-Architekturen durch eine virtuelle, gröber granulare Zuordnungsarchitektur beschrieben. Durch die Artefakte dieser zusätzlichen Alignment-Architektur werden die direkten Punkt-zu-Punkt-Zuordnungen zwischen den fachlichen Artefakten und den IT-Artefakten durch indirekte Zuordnungen über gröber granulare fachliche Services, Applikationen und Domänen ersetzt. Dadurch wird gesamthaft eine indirekte m:n-Zuordnung geschaffen, die im Hinblick auf Änderungen fachlicher Artefakte oder Änderungen der IT-Artefakte effizienter ist, weil sie viele Änderungen „puffert“.

Dieser Lösungsansatz geht in verschiedener Hinsicht über bestehende Ansätze hinaus:

- Klassische Middleware, Enterprise Application Integration und andere m: n-IT-Zuordnungsmechanismen sind immer auf Artefakte des gleichen Typs beschränkt. Erst eine zusätzliche Architekturebene mit dedizierten, neuen Zuordnungsartefakten ist in der Lage, auch Artefakte verschiedener Typen (z. B. fachliche Artefakte und IT-Artefakte) zu entkoppeln.

- Auch serviceorientierte Architekturen wirken zwar entkoppelnd und flexibilitätssteigernd, beschränken sich aber ebenfalls auf die verknüpfende Integration gleichartiger Artefakte. Allein durch Serviceorientierung auf Fachund IT-Seite wird die in Abschnitt 1 skizzierte Alignment-Problematik nicht gelöst. Hier schafft erst eine systematische Verknüpfung über die Architekturebenen hinweg Lösungspotenziale. Gleichwohl wird davon ausgegangen, dass die Elementarartefakte der Alignment-Architektur serviceorientiert strukturiert werden. Dies ist allein schon deshalb sinnvoll, um eine konsistente Verknüpfung mit den zu integrierenden, im Idealfall auch serviceorientiert strukturierten Artefakten zu ermöglichen.

- Referenz-Informationssystemarchitekturen (wie z. B. das Handels-H-Modell oder VAA) stellen bewährte Lösungen mit einem bestimmten Wiederverwendungspotenzial und einem bestimmten Empfehlungscharakter dar, in denen aufgrund des Zeitpunktbezugs Alignment-Probleme ausgeblendet sind. IT/ Business Alignment ist deshalb ein zentrales Problem des Informationsmanagements, weil eine mühsam (z. B. durch Umsetzung eines Referenzmodells) geschaffene Konsistenz zwischen fachlicher Architektur und IT-Architektur aufgrund ständiger Anpassungen auf beiden Seiten schnell wieder verloren geht. Die hier vorgeschla- gene Alignment-Architektur adressiert primär diesen dynamischen Aspekt. Bei vollkommener Stabilität der fachlichen Architektur und der IT-Architektur schafft sie keinen Mehrwert. Wie in Abschnitt 1 dargelegt wurde, orientiert sich das Vorgehen in diesem Beitrag am Prozessmodell gestaltungsorientierter Informationssystem-Forschung (Rossi u. Sein 2003). Darum wurde nach der Konstruktion des generischen Lösungsvorschlags die Bottom-up-Konstruktion virtueller Alignment-Artefakte am Beispiel der Domänenbildung prototypisch implementiert und in einer Fallstudie evaluiert.

Weiteren Forschungsbedarf sehen wir in der Integration und ggf. Ergänzung der bestehenden Ansätze für den Entwurf der verschiedenartigen Artefakte der Alignment-Architektur. Die integrierten Entwurfsstrategien können zum einen, wie hier vorgestellt, einem Bottom-up-Ansatz auf Basis der bestehenden Architektur folgen. Alternativ (und als Konsistenzprüfung) ist es denkbar, einem Top-downAnsatz deduktiv durch die Ableitung der Alignment-Artefakte aus den Grobstrukturen der fachlichen Architektur und der IT-Architektur zu folgen. Die Herausforderung besteht dabei in der Abbildung der jeweils unterschiedlichen Gestaltungsziele der angrenzenden Architekturen (Prozesseffektivität und -effizienz auf fachlicher Ebene vs. Wiederverwendung, Skalierbarkeit und Performanz auf IT-Ebene, vgl. Abschnitt 2) auf das Alignment-Ziel. In einem originären Top-down-Ansatz wiederum können die Ergebnisse von modellgetriebenen Bottom-up-Analysen, wie hier vorgestellt, eine wertvolle weitere Perspektive für die Gestaltung der Alignment-Architektur bieten. Das hier vorgestellte Bottom-up-Vorgehen basiert auf einer Analyse der fachlichen Ist-Architektur und der IT-Ist-Architektur. Die „puffernde"Wirkung der Alignment-Architektur kann jedoch durch eine konsequentere Implementierung von „loose coupling“ in den entkoppelten Architekturen erheblich verstärkt werden. Weiterer Forschungsbedarf besteht deshalb in der Entwicklung eines Ansatzes, mit welchem sich aus der Bottom-up-Analyse der Ist-Architekturen Gestaltungshinweise für verbesserte SollArchitekturen ableiten lassen. Als Indikator für die Bewertung der Entkopplung der Architekturen lässt sich dann beispielsweise die Anzahl der entfernten Kanten pro gebildetem Cluster heranziehen. 


\section{Zusammenfassung / Abstract}

Stephan Aier, Robert Winter

\section{Virtuelle Entkopplung von fachlichen und IT-Strukturen für das IT/ Business Alignment - Grundlagen, Architekturgestaltung und Umsetzung am Beispiel der Domänenbildung}

Die Integration von Business und IT ist eines der Kernthemen der Wirtschaftsinformatik. Die direkte Punkt-zu-Punkt-Kopplung von fachlichen und IT-Artefakten wird den Anforderungen an die Änderbarkeit von fachlichen und IT-Architekturen nicht gerecht. Die Informatik hat beispielsweise mit der Einführung der "Dreiebenen-Datenbankarchitektur" eine virtuelle Zuordnungsarchitektur geschaffen, die Elemente der externen und internen Ebene über die konzeptuelle Ebene entkoppelt. Dieser Beitrag entwirft analog dazu eine Alignment-Architektur für das IT/Business Alignment und stellt als entsprechende Artefakte fachliche Services, Applikationen und Domänen vor. Als Alternative zu verbreiteten Top-down-Ansätzen für die Bildung von fachlichen Services, Applikationen und Domänen wird am Beispiel der Domänenbildung ein Bottom-up-Ansatz vorgeschlagen und anhand einer Fallstudie aus der Telekommunikationsindustrie demonstriert. Schlüsselwörter: Integration, Entkopplung, IT/Business Alignment

\section{Virtual Decoupling for IT/Business Alignment - Conceptual Foundations, Architecture Design and Implementation Example}

IT/business alignment is one of the main topics of information systems research. If IT artifacts and business-related artifacts are coupled point-to-point, however, complex architectures become unmanageable over time. In computer science, concepts like the ANSI/SPARC three-level database architecture propose an architecture layer which decouples external views on data and the implementation view of data. In this paper, a similar approach for IT/business alignment is proposed. The proposed alignment architecture is populated by enterprise services as elementary artifacts. Enterprise services link software components and process activities. They are aggregated into applications and subsequently into domains for planning/design and communication purposes. Most design approaches for the construction of enterprise services, applications and domains are top-down, i. e. stepwise decompose complex artifacts. As an alternative which takes into account coupling semantics, we propose a bottom-up approach which is demonstrated for the identification of domains. Our approach is evaluated using a telecommunications equipment case study.

Keywords: Integration, Decoupling, IT/business alignment
Zur Wahrung der entkoppelnden Wirkung der virtuellen Alignment-Architektur wird explizit keine Konsistenz zwischen den hierarchischen Verfeinerungen der fachlichen Architektur, der Alignment-Architektur und der IT-Architektur gefordert. Für die Hierarchisierung der Artefakte der Alignment-Architektur selbst sind jedoch Gestaltungsvorschriften zu entwickeln und zu überprüfen. Insbesondere ist die Frage zu beantworten, ob die Hierarchiebildung ausschließlich mit 1:n-Zuordnungen oder aber auch mit m:nZuordnungen effektiv und effizient ist.

Unsere Analyse der Zuordnungsmechanismen schließt bislang nur die strukturellen Aspekte dieser Mechanismen ein. In weiterführenden Arbeiten müssen zusätzlich auch das aufeinander bezogene Verhalten der fachlichen, IT- und der Alignment-Architektur und somit der Schnittstellencharakter der Alignment-Artefakte berücksichtigt werden.

Schließlich muss in weiteren Arbeiten eine „Infrastruktur“ für die Entwicklung und den Betrieb einer Alignment-Architektur entwickelt werden. So sind beispielsweise Modell-Repositorys bereitzustellen, in welchen zum Zweck der Entwicklung der Alignment-Architektur die relevanten Fach- und IT-Architekturen abgebildet werden. Zum Zweck des Betriebs der Alignment-Architektur müssen in den Repositorys ebenfalls die Alignment-Artefakte mit ihren Beziehungen untereinander sowie ihren Beziehungen zu den Fach- und IT-Architekturen abgebildet werden. Solche Modell-Repositorys schaffen die notwendige Transparenz für die Entwicklung der Alignment-Architektur und für die anschließende Weiterentwicklung der angrenzenden Architekturen.

Die so geschaffene Transparenz ist gleichzeitig die Voraussetzung für die Bewertung der Effektivität und Effizienz dieses Architekturansatzes im Zeitverlauf. So kann beispielsweise bewertet werden, bei welcher Komplexität und welcher Änderungsfrequenz das Hinzufügen zusätzlicher Artefakte der AlignmentArchitektur und somit die Erhöhung der Komplexität messbar durch eine Verringerung der Anzahl propagierter Änderungen überkompensiert werden. 


\section{Literatur}

Aier S (2006) How clustering enterprise architectures helps to design service oriented architectures. In: Proceedings of the IEEE international conference on services computing (SCC'06). IEEE Computer Society, Los Alamitos, S 269272

Ashby WR (1981) The set theory of mechanism and homeostasis. In: Conant R (Hrsg) Mechanisms of intelligence: Ross Ashby's Writings on Cybernetics. Intersystems, Seaside, S 21-49

Bath U, Herr M (2004) Implementation of a service oriented architecture at Deutsche Post MAIL. In: Aier S, Schönherr M (Hrsg) Enterprise Application Integration - Serviceorientierung und nachhaltige Architekturen. GITO, Berlin, S 279-297

Buxmann P, König W (1998) Das Standardisierungsproblem: Zur ökonomischen Auswahl von Standards in Informationssystemen. WIRTSCHAFTSINFORMATIK 40(2):122-129

Buxmann P, Weitzel T, von Westarp F, König W (1999) The standardization problem: an economic analysis of standards in information systems. In: Proceedings of the 1st IEEE conference on standardisation and innovation in information technology SIIT‘99, S 157-162

Date CJ (2000) An introduction to database systems, 7. Aufl. Addison Wesley, Reading

Devlin B (1997) Data warehouse: from architecture to implementation. Addison Wesley, Reading

Dietrich J (2008) Nutzung von Modellierungssprachen und -methodologien standardisierter B2B-Architekturen für die Integration unternehmensinterner Geschäftsprozesse. Gito, Berlin

Frank H, Bobrik A, Haarländer N (2007) Vorgehensmodell der Systemanalyse. In: Krallmann H, Schönherr M, Trier M (Hrsg) Systemanalyse im Unternehmen - Prozessorientierte Gestaltung von Unternehmen, 5. Aufl. Oldenbourg, München, S 135-186

Freeman LC (1977) A set of measures of centrality based upon betweenness. Sociometry 40(1):35-41

Frese E (1993) Geschäftssegmentierung als organisatorisches Konzept. ZfbF 45(12):999-1024

Fritsch M, Wein T, Ewers H-J (2005) Marktversagen und Wirtschaftspolitik: mikroökonomische Grundlagen staatlichen Handelns, 6. Aufl. Vahlen, München

Girvan M, Newman MEJ (2002) Community structure in social and biological networks. Proceedings of the National Academy of Science 99(12):7821-7826

Glassman RB (1973) Persistence and loose coupling in living systems. Behavioral Science 18(2):83-98

Hagen C (2003) Integrationsarchitektur der Credit Suisse. In: Aier S, Schönherr M (Hrsg) Enterprise Application Integration - Flexibilisierung komplexer Unternehmensarchitekturen. GITO, Berlin, S 61-81

Henderson JC, Venkatraman N (1993) Strategic alignment: leveraging information technology for transforming organizations. IBM Systems
Journal 32(1):4-16

Herdzina K (2005) Einführung in die Mikroökonomik, 10. Aufl. Vahlen, München

Heutschi R, Legner C (2007) SOA adoption in practice - findings from early SOA implementations. In: Proceedings of the 15th European conference on information Systems "Relevant rigour - rigorous relevance"

IBM (1984) Business systems planning - information systems planning guide, IBM-Form GE200527-4, 4. Aufl. IBM, Atlanta

IMG (1999) PROMET STP: Methodenhandbuch für die System- und Technologieplanung, Release 1.0. IMG AG, St. Gallen

Inmon WH (1996) Building the data warehouse, 2. Aufl. Wiley, New York

Kaib M (2002) Enterprise Application Integration - Grundlagen, Integrationsprodukte, Anwendungsbeispiele. DUV, Wiesbaden

Kantzenbach E (1967) Die Funktionsfähigkeit des Wettbewerbs, 2. Aufl. Vandenhoeck \& Ruprecht, Göttingen

Katz ML, Shapiro C (1994) Systems competition and network effects. J Economic Perspectives 8(2):93-115

Kerber W (2007) Wettbewerbspolitik. In: Bender D, Berg H, Cassel D et al (Hrsg) Vahlens Kompendium der Wirtschaftstheorie und Wirtschaftspolitik, Vol. 2. Vahlen, München, S 369434

Kollmann T (2001) Virtuelle Marktplätze: Grundlagen - Management - Fallstudie. Vahlen, München

Linthicum DS (2000) Enterprise application integration. AWL Direct Sales, Reading

Luftman JN (2005) Key issues for IT executives 2004. In: MIS Quarterly Executive 4(2):269-285

Luhmann N (1991) Soziologie des Risikos. de Gruyter, Berlin

Luhmann N (2002) Einführung in die Systemtheorie. Carl Auer, Heidelberg

Meffert JPH (1993) Standards als Integrationsinstrument in der Computer- und Kommunikationsindustrie: wettbewerbsstrategische Bedeutung und Durchsetzung. Universitätsverlag, Konstanz

Mertens $P$ (2007) Integrierte Informationsverarbeitung 1, 16. Aufl. Gabler, Wiesbaden

Newman MEJ (2004) Analysis of weighted networks. Phys. Rev. E 70 (056131)

O'Madadhain J, Fisher D, Smyth P, White S, Boey Y-B (2005) Analysis and visualization of network data using JUNG. J Statistical Software (Preprint)

Perrow C (1999) Normal accidents: living with high-risk technologies. Princeton University Press, Princeton

Ricardo D (1830) On the principles of political economy and taxation, 2. Aufl. Bell, Washington

Rosemann M (1999) Gegenstand und Aufgaben des Integrationsmanagements. In: Scheer A-W, Rosemann M, Schütte R (Hrsg) Integrationsmanagement. Institut für Wirtschaftsinformatik, Universität Münster, S 5-18

Rossi M, Sein MK (2003) Design research workshop: a proactive research approach. http:// www.cis.gsu.edu/ emonod/epistemology/
Sein\%20and\%20Rossi\%20-

\%20design\%20research\%20-\%20IRIS.pdf. Abruf am 2008-08-14

Schelp J, Winter R (2007) Towards a methodology for service construction. In: Proceedings of the 40th Hawaii international conference on systems sciences (HICSS-40). IEEE Computer Society, Los Alamitos, S64a

Schelp J, Winter R (2008) Entwurf von Anwendungssystemen und Entwurf von Enterprise Services - Ähnlichkeiten und Unterschiede. WIRTSCHAFTSINFORMATIK 50(1):6-15

Shapiro C (2001) Setting compatibility standards: cooperation or collusion? In: Cooper Dreyfuss $\mathrm{R}$, Leenheer Zimmerman DL, First H (Hrsg) Expanding the bounds of intellectual property. University Press, Oxford, S 81-101

Smith A (1776) An inquiry into the nature and causes of the wealth of nations. Strahan and Cadell, London

Stünzer L (1996) Systemtheorie und betriebswirtschaftliche Organisationsforschung. Duncker \& Humblot, Berlin

Vogler P (2003) Prozess- und Systemintegration: Umsetzung des organisatorischen Wandels in Prozessen und Informationssystemen. Habilitation, Universität St. Gallen

Weiber R, Kollmann T (1998) Competitive advantages in virtual markets - perspectives of „information-based marketing" in cyberspace. Eur J Marketing 32(7/8):603-615

Weick KE (1976) Educational organizations as loosely coupled systems. Administrative Science Quarterly 21(1):1-19

Winter R (2003) An architecture model for supporting application integration decisions. In: Ciborra C et al (Hrsg) Proc. 11th European Conference on Information Systems

Winter R (2008) Business Engineering - Betriebswirtschaftliche Konstruktionslehre und ihre Anwendung in der Informationslogistik. In: Dinter B, Winter R (Hrsg) Integrierte Informationslogistik. Springer, Heidelberg, S 17-38

Winter R, Schelp J (2006) Dienstorientierte Architekturgestaltung auf unterschiedlichen $\mathrm{Ab}$ straktionsebenen. In: Reussner R, Hasselbring W (Hrsg) Handbuch der Software-Architektur. dpunkt, Heidelberg, S 229-242

Wu F, Huberman BA (2004) Discovering communities in linear time: a physics approach. Eur Phys J B 38(2):331-338 